Muhammad Jamil* and Abdul Haleem

\title{
MHD fractionalized Jeffrey fluid over an accelerated slipping porous plate
}

https://doi.org/10.1515/nleng-2020-0015

Received Jun 10, 2019; accepted Mar 11, 2020.

\begin{abstract}
The primary target of this paper is to obtain the analytic solutions for the incompressible unsteady flow of fractionalized MHD Jeffrey fluid over an accelerating porous plate with linear slip effect is assumed between fluid and the plate. The governing equations of Jeffrey fluid are developed by fractional calculus approach. The velocity distribution and its corresponding shear stress both are obtained in terms of generalized $\mathbf{M}$-function by using Laplace transform technique and considering all initial and boundary conditions. We have also discussed that obtained results of fractionalized MHD Jeffrey fluid for different cases for instance, with and without slip effects, with and without MHD and porosity effects. The influence of the different parameters affected on the flow characteristic is deliberated with the help of graphs. Finally, the analysis among different fluid models exhibits by graphical illustrations.
\end{abstract}

Keywords: Jeffrey fluid, fractional calculus, MHD, slip effects, porosity, unsteady flow, velocity distribution, shear stress, Laplace transforms technique, M-function.

\section{Introduction}

It is investigated from the last few decades, that many researchers are much interested in Non-Newtonian fluids. The explanation behind such quickening interest is because of the extensive scope of utilization of nonNewtonian fluids. The non-Newtonian fluids have several applications in different regions for an instant, geophysics and biological sciences, petroleum, and chemical industries. As we know that all non-Newtonian fluids

*Corresponding Author: Muhammad Jamil, Department of Mathematics, NED University of Engineering \& Technology, Karachi-75270, Pakistan. Tel. +92-3136618192, Fax: +92-021-99230602,

E-mail: jqrza@neduet.edu.pk, jqrza26@yahoo.com

Abdul Haleem, Department of Mathematics, NED University of Engineering \& Technology, Karachi-75270, Pakistan have both the properties of viscosity as well as elasticity. Non-Newtonian fluids have many examples, such as honey, ketchup, oils, toothpaste and paints, liquid polymers and asphalt are represented some remarkable phenomena. These fluids are several interesting applications and also used in our life. Many researchers have been proven that such type of fluids has a non-linear relationship between shear rate and shear stress and these are not only significant for academia but also important for industries like polymer processing and the making of papers and foods. It is observed that the flow of Newtonian models is explained in a single constitutive equation but nonNewtonian fluids flow cannot be represented by a single constitutive equation. In general, the rheological properties of liquids are indicated by their supposed constitutive conditions. Further, it is noticed that fluids are satisfied Newtonian law of inner friction that shear stress is proportional to the viscosity of the fluid gradient and nonnewtonian fluids do not satisfies the law of inner fraction. The governing equations of flow the non-Newtonian fluids are more complex than Navier-Stokes equations[13]. In general Non-Newtonian fluid is classified into three different classes namely, integral type, differential type, and rate type. The Jeffrey fluid model is considered in the present study, this type of fluid shows the properties of the ratio of relation to retardation time. It has been proven that the non-Newtonian fluid flow in the presence as well as in absence of magnetic field has many applications in different fields for an instant, the handling of biological fluids, plasma, mercury amalgams, liquid metals and alloys, blood and electromagnetic propulsion $[4,5]$.

It is notified that Jeffrey fluid is a special kind of nonNewtonian fluid. In different models of non-Newtonian fluids, it is shown that Jeffrey fluid model is one of the significant models which defined the best explanation of the rheological viscoelastic fluids, it is used the time derivative rather than convected derivative [6 - 11]. It is exhibited that Jeffrey fluid models in nature are defined in linear viscoelastic fluids. As we know that in polymer industries Jeffrey fluids have many applications, dilute polymer is one of them discussed by Farooq et al. [12] and Ara et al. [13]. The important and appropriate role of Jeffrey fluid models is found in biological and fluid mechanics due to 
viscoelastic behavior, it has been successfully used in the blood flow model. Jeffrey fluid is interrelated with Newtonian fluid and Maxwell fluid as a special case [14].

It is found in physiology, the magnetohydrodynamic field have many applications such as (i): blood a bio magnetic fluid is created in the presence of hemoglobin molecule (ii): magnetic resonance imaging (iii): magnetic particles and magmatic devices are used as a drug carriers [15]. Tripathi et al. [16] investigated the influence of MHD on finite length of cylindrical tube in Jeffrey fluid flow. Das [17] and Afridi et al. [18] studied that in the existence of heat transform and slip impacts on MHD Jeffrey fluid flow. Jamil et al. [19] investigated the influence of MHD in Oldroyed-B fluid flow over a plane. Mohanty et $a l$. [20] have analyzed the influence of MHD on Jeffrey fluid flow and also discussed the effects of chemical reaction on MHD flow of Jeffrey fluid. Kashyap et al. [21] studied MHD slip flow of chemically reacting upper convected Maxwell fluid through a dilating channel with heat source/sink. Hayat et al. [22] discovered in channel MHD flow of Jeffrey fluid in series solutions. Zheng et al. [23] discussed the influence of slip on MHD of a generalized oldroyd- B fluid flow and determined analytic solution. Sharma et al. [24] studied viscous dissipation and thermal radiation effects in MHD flow of Jeffrey nanofluid through impermeable surface with heat generation/absorption. Jamil et al. [25] and Yaqing [26] are investigated the influence of first order slip condition of fractional Maxwell fluid and found exact solutions of velocity field and shear stress. Further MHD and slip influence on Jeffrey fluid with fractional calculus have been discussed by various authors in [27 - 32].

The main purpose of this present paper is to investigate the influence of MHD fractionalized Jeffrey fluids over an accelerated slipping porous plate. In this present investigation, classical Jeffrey model is converted into fractionalized model by using Caputo fractional operator. The governing equations are transformed into fractionalized MHD Jeffrey fluid by taking fractional parameter $\alpha$. To find analytic solutions for velocity field and shear stress, we have used Laplace transform on fractionalized governing equations of Jeffrey fluid. Here we have considered MHD and porous plate. The generalized solutions are determined and express in series form as well as in term of the generalized $\mathbf{M}$-function satisfying all initial and boundary conditions. From the obtained general solutions the related solutions for ordinary Jeffrey fluid can be determined as as special cases of general solutions. The second grade and Newtonian fluid solutions can also be analyzed as limiting cases of fractional and ordinary Jeffrey fluids. More precisely, the solutions for without slip condition for fractionalized and ordinary Jeffrey fluid are also determined as a special cases and they are related with obtained pervious results in literature. Finally, the impacts of different materials parameters, slip and fractional parameters on the movement of ordinary Jeffrey fluid and fractionalized fluid are represented by graphical illustration. The difference is also highlighted among the fractionalized Jeffrey fluid, ordinary Jeffrey fluid, Newtonian fluid with MHD and porous and simple Newtonian fluids graphically.

\section{Fractionalized governing equations}

In the following problem we consider a velocity field and additional stress of the form of

$$
\mathbf{V}=(u(y, t), 0,0), \mathbf{S}=\mathbf{S}(y, t),
$$

where $u$ is the velocity in $\mathrm{x}$-direction. The continuity equation has been satisfied, at $t=0$ the rest of fluid, constitutive equations of Jeffrey fluid and equation of motion provide that $S_{y y}=S_{y z}=S_{z z}=0$ and the meaningful equations

$$
\begin{aligned}
& \left(1+\lambda_{1}\right) \frac{\partial u(y, t)}{\partial t}=v\left(1+\lambda_{2} \frac{\partial}{\partial t}\right) \frac{\partial^{2} u(y, t)}{\partial y^{2}} \\
& -v K\left(1+\lambda_{2} \frac{\partial}{\partial t}\right) u(y, t)-H\left(1+\lambda_{1}\right) u(y, t), \\
& \left(1+\lambda_{1}\right) \tau(y, t)=\mu\left(1+\lambda_{2} \frac{\partial}{\partial t}\right) \frac{\partial u(y, t)}{\partial y},
\end{aligned}
$$

where $\tau=S_{x y}$ is the tangential stress, $v=\mu / \rho$ is the kinematic viscosity, $\mu$ is the dynamic viscosity, $\rho$ is the density of the fluid and $K=\frac{\phi}{k}$ and $H=\frac{\sigma B_{o}^{2}}{\rho}$ are magnetic and porosity constants, where $\phi$ is the porosity and $\kappa$ is the permeability of the porous medium, $B_{0}$ is the magnitude of applied magnetic field and $\sigma$ is the electrically conductively of fluid. It is important to note that by putting $\lambda_{1}=0$, and $\lambda_{1}=\lambda_{2}=0$ in above equations we recover the governing equations of second grade and Newtonian fluids.

The governing equations of an incompressible MHD Jeffrey fluid with fractional derivatives, performing the same motion in the absence of pressure gradient, are

$$
\begin{gathered}
\left(1+\lambda_{1}\right) \frac{\partial u(y, t)}{\partial t}=v\left(1+\lambda_{2}^{\alpha} D_{t}^{\alpha}\right) \frac{\partial^{2} u(y, t)}{\partial y^{2}} \\
-v K\left(1+\lambda_{2}^{\alpha} D_{t}^{\alpha}\right) u(y, t)-H\left(1+\lambda_{1}\right) u(y, t), \\
\tau=\frac{\mu}{\left(1+\lambda_{1}\right)}\left(1+\lambda_{2}^{\alpha} D_{t}^{\alpha}\right) u(y, t),
\end{gathered}
$$


where $\alpha$ is the fractional parameter, and the fractional differential operator so called Caputo fractional operator $D_{t}^{\alpha}$ defined by [32]

$$
D_{t}^{\alpha} f(t)= \begin{cases}\frac{1}{\Gamma(1-\alpha)} \int_{0}^{t} \frac{f^{\prime}(\tau)}{(t-\tau)^{\alpha}} d \tau, & 0<\alpha<1 ; \\ \frac{d f(t)}{d t}, & \alpha=1 .\end{cases}
$$

\section{Formulation of the problem \& solutions}

Consider the incompressible fractionalized MHD Jeffrey fluid models covering the space lying over an accelerating porous plate. It is exhibited that the velocity field is measured into $(y, t)$ and in the direction of $y$-axis depict perpendicular to the plate. In starting the velocity distribution of fluid movement will be zero. It is considered at time $t=0^{+}$the plate moves with accelerated velocity $A t^{p}$. Due to force stress over the fluid, gradually it starts to move above the plate. Its velocity is of the form $(1)_{1}$ while the governing equations are given by Eqs. (4) and (5). The appropriate initial and boundary conditions are

$$
\begin{gathered}
u(y, 0)=0, \tau(y, 0)=0, \quad y>0, \\
u(0, t)=A H(t) \mathrm{t}^{p}+\left.\theta \frac{\partial u(y, t)}{\partial y}\right|_{y=0} ; t, p \geq 0 .
\end{gathered}
$$

The function $\mathrm{H}(\mathrm{t})$ is called Heaviside and $\theta$ is called slip parameter. If $\theta=0$, then considered that no boundary slip condition can be calculated. If slip parameter $\theta$ is limiting values, at the wall slip influence is accrued, it is observed that influence fluid flow depends on length scale. Moreover, the natural conditions are employed

$$
u(y, t) \text { and } \frac{\partial u(y, t)}{\partial y} \rightarrow 0 \text { as } y \rightarrow \infty \text { and } t>0 .
$$

\subsection{Investigation the solution of Velocity distribution field}

Employing Laplace transform, to Eq. (4) and having in mind initial and boundary conditions in Eqs. (6) and (7), we get

$$
\frac{\partial^{2} \bar{u}(y, q)}{\partial y^{2}}-\frac{(H+q)\left(1+\lambda_{1}\right)+v K\left(1+\lambda_{2}^{\alpha} q^{\alpha}\right)}{v\left(1+\lambda_{2}^{\alpha} q^{\alpha}\right)} \bar{u}(y, q)=0,
$$

subject to boundary condition

$$
\bar{u}(0, q)=\frac{A p !}{q^{p+1}}+\left.\theta \frac{\partial \bar{u}(y, q)}{\partial y}\right|_{y=0},
$$

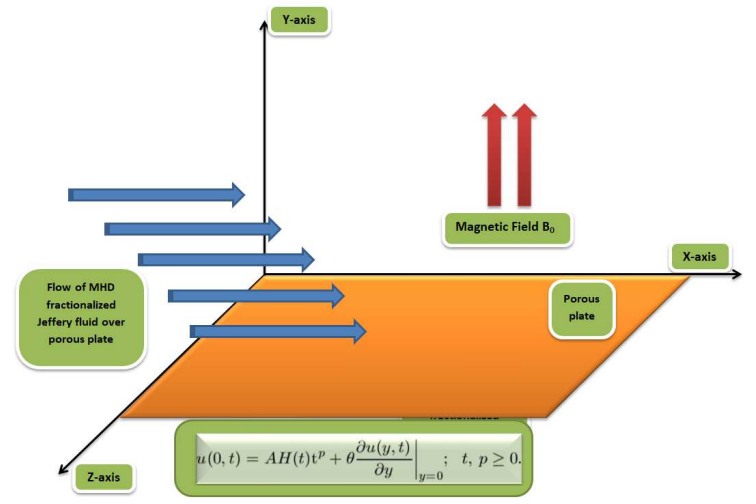

Figure 1: Flow of MHD fractionalized Jeffrey fluid over an accelerated slipping porous plate

and natural condition

$$
\bar{u}(y, q), \quad \frac{\partial \bar{u}(y, q)}{\partial y} \rightarrow 0 \text { as } y \rightarrow \infty,
$$

where $\bar{u}(y, q)$ is called pre-image of $u(y, t)$ and $q$ is known as transform parameter. Solving Eqs.(9) by utilizing natural conditions in Eq. (11), we get

$$
\begin{aligned}
& \bar{u}(y, q)=\frac{A p !}{q^{p+1}\left\{1+\theta\left[\frac{\left(1+\lambda_{1}\right)(q+H)+v K\left(1+\lambda_{2}^{\alpha} q^{\alpha}\right)}{v\left(1+\lambda_{2}^{\alpha} q^{\alpha}\right)}\right]^{\frac{1}{2}}\right\}} \\
& \times \exp \left\{-\left[\frac{\left(1+\lambda_{1}\right)(q+H)+v K\left(1+\lambda_{2}^{\alpha} q^{\alpha}\right)}{v\left(1+\lambda_{2}^{\alpha} q^{\alpha}\right)}\right]^{\frac{1}{2}} y\right\} .
\end{aligned}
$$

To avoid the complex computations of residuals and contours integrals, now we will simplify Eq. (12) and represents in-terms of series form we get velocity field in the form of series

$\bar{u}(y, q)=$

$\frac{A p !}{q^{p+1}}+A p ! \sum_{b=1}^{\infty}\left(\frac{-\theta}{\sqrt{v}}\right)^{b} \sum_{c=0}^{\infty} \frac{(-v K)^{c}}{c !} \sum_{d=0}^{\infty} \frac{(-H)^{d}}{d !}\left(\frac{1+\lambda_{1}}{\lambda_{2}^{\alpha}}\right)^{\left(\frac{b}{2}-c\right)}$

$\times \sum_{e=0}^{\infty} \frac{\Gamma\left(-\frac{b}{2}+c\right) \Gamma\left(-\frac{b}{2}+c+d\right) \Gamma\left(\frac{b}{2}-c+e\right)\left(-\lambda_{2}^{\alpha}\right)^{-e}}{e ! \Gamma\left(\frac{-b}{2}\right) \Gamma\left(-\frac{b}{2}+c\right) \Gamma\left(\frac{b}{2}-c\right)}$

$\times \frac{1}{q^{-\left(\frac{b}{2}-c\right)(1-\alpha)+d+\alpha e+p+1}}+A p ! \sum_{a=1}^{\infty} \frac{1}{a !}\left(\frac{-y}{\sqrt{v}}\right)^{a} \sum_{b=0}^{\infty}\left(\frac{-\theta}{\sqrt{v}}\right)^{b}$

$\times \sum_{c=0}^{\infty} \frac{(-v K)^{c}}{c !} \sum_{d=0}^{\infty} \frac{(-H)^{d}}{d !}\left(\frac{1+\lambda_{1}}{\lambda_{2}^{\alpha}}\right)^{\left(\frac{a+b}{2}-c\right)}$

$\times \sum_{e=0}^{\infty} \frac{\Gamma\left(-\frac{a+b}{2}+c\right) \Gamma\left(-\frac{a+b}{2}+c+d\right) \Gamma\left(\frac{a+b}{2}-c+e\right)\left(-\lambda_{2}^{\alpha}\right)^{-e}}{e ! \Gamma\left(-\frac{a+b}{2}\right) \Gamma\left(-\frac{a+b}{2}+c\right) \Gamma\left(\frac{a+b}{2}-c\right)}$

$\times \frac{1}{q^{-\left(\frac{a+b}{2}-c\right)(1-\alpha)+d+\alpha e+p+1}}$. 
Applying discrete inverse Laplace transform to Eq. (13), we obtain velocity field

$$
\begin{aligned}
u(y, t)= & A H(t) t^{p}+A H(t) p ! \sum_{b=1}^{\infty}\left(\frac{-\theta}{\sqrt{v}}\right)^{b} \sum_{c=0}^{\infty} \frac{(-v K)^{c}}{c !} \sum_{d=0}^{\infty} \frac{(-H)^{d}}{d !}\left(\frac{1+\lambda_{1}}{\lambda_{2}^{\alpha}}\right)^{\left(\frac{b}{2}-c\right)} \\
& \times \sum_{e=0}^{\infty} \frac{\Gamma\left(-\frac{b}{2}+c\right) \Gamma\left(-\frac{b}{2}+c+d\right) \Gamma\left(\frac{b}{2}-c+e\right)\left(-\lambda_{2}^{\alpha}\right)^{-e}}{e ! \Gamma\left(\frac{-b}{2}\right) \Gamma\left(-\frac{b}{2}+c\right) \Gamma\left(\frac{b}{2}-c\right)} \frac{t^{-\left(\frac{b}{2}-c\right)(1-\alpha)+d+\alpha e+p}}{\Gamma\left(-\left(\frac{b}{2}-c\right)(1-\alpha)+d+\alpha e+p+1\right)} \\
& +A H(t) p ! \sum_{a=1}^{\infty} \frac{1}{a !}\left(\frac{-y}{\sqrt{v}}\right)^{a} \sum_{b=0}^{\infty}\left(\frac{-\theta}{\sqrt{v}}\right)^{b} \sum_{c=0}^{\infty} \frac{(-v K)^{c}}{c !} \sum_{d=0}^{\infty} \frac{(-H)^{d}}{d !}\left(\frac{1+\lambda_{1}}{\lambda_{2}^{\alpha}}\right)^{\left(\frac{a+b}{2}-c\right)} \\
\times & \sum_{e=0}^{\infty} \frac{\Gamma\left(-\frac{a+b}{2}+c\right) \Gamma\left(-\frac{a+b}{2}+c+d\right) \Gamma\left(\frac{a+b}{2}-c+e\right)\left(-\lambda_{2}^{\alpha}\right)^{-e}}{e ! \Gamma\left(-\frac{a+b}{2}\right) \Gamma\left(-\frac{a+b}{2}+c\right) \Gamma\left(\frac{a+b}{2}-c\right)} \frac{t^{-\left(\frac{a+b}{2}-c\right)(1-\alpha)+d+\alpha e+p}}{\Gamma\left(-\left(\frac{a+b}{2}-c\right)(1-\alpha)+d+\alpha e+p+1\right)} .
\end{aligned}
$$

Equvalently

$$
\begin{aligned}
u(y, t)= & A H(t) t^{p}+A H(t) p ! \sum_{b=1}^{\infty}\left(\frac{-\theta}{\sqrt{v}}\right)^{b} \sum_{c=0}^{\infty} \frac{(-v K)^{c}}{c !} \sum_{d=0}^{\infty} \frac{(-H)^{d}}{d !}\left(\frac{1+\lambda_{1}}{\lambda_{2}^{\alpha}}\right)^{\left(\frac{b}{2}-c\right)} t^{-\left(\frac{b}{2}-c\right)(1-\alpha)+d+p} \\
& \times \sum_{e=0}^{\infty} \frac{\Gamma\left(-\frac{b}{2}+c\right) \Gamma\left(-\frac{b}{2}+c+d\right) \Gamma\left(\frac{b}{2}-c+e\right)}{e ! \Gamma\left(\frac{-b}{2}\right) \Gamma\left(-\frac{b}{2}+c\right) \Gamma\left(\frac{b}{2}-c\right)} \frac{\left(\frac{-t^{\alpha}}{\lambda_{2}^{\alpha}}\right)^{e}}{\Gamma\left(-\left(\frac{b}{2}-c\right)(1-\alpha)+d+\alpha e+p+1\right)} \\
+ & A H(t) p ! \sum_{a=1}^{\infty}\left(\frac{-y}{\sqrt{v}}\right)^{a} \sum_{b=0}^{\infty}\left(\frac{-\theta}{\sqrt{v}}\right)^{b} \sum_{c=0}^{\infty} \frac{(-v K)^{c}}{c !} \sum_{d=0}^{\infty} \frac{(-H)^{d}}{d !}\left(\frac{1+\lambda_{1}}{\lambda_{2}^{\alpha}}\right)^{\left(\frac{a+b}{2}-c\right)} t^{-\left(\frac{a+b}{2}-c\right)(1-\alpha)+d+p} \\
& \times \sum_{e=0}^{\infty} \frac{\Gamma\left(-\frac{a+b}{2}+c\right) \Gamma\left(-\frac{a+b}{2}+c+d\right) \Gamma\left(\frac{a+b}{2}-c+e\right)}{e ! \Gamma\left(-\frac{a+b}{2}\right) \Gamma\left(-\frac{a+b}{2}+c\right) \Gamma\left(\frac{a+b}{2}-c\right)} \frac{\left(\frac{-t^{\alpha}}{\lambda_{2}^{\alpha}}\right)^{e}}{\Gamma\left(-\left(\frac{a+b}{2}-c\right)(1-\alpha)+d+\alpha e+p+1\right)} .
\end{aligned}
$$

Now represents the above velocity field in terms of generalized $\mathbf{M}$-function, we get

$$
\begin{aligned}
u(y, t)= & A H(t) t^{p}+A H(t) p ! \sum_{b=1}^{\infty}\left(\frac{-\theta}{\sqrt{v}}\right)^{b} \sum_{c=0}^{\infty} \frac{(-v k)^{c}}{c !} \sum_{d=0}^{\infty} \frac{(-H)^{d}}{d !}\left(\frac{1+\lambda_{1}}{\lambda_{2}^{\alpha}}\right)^{\left(\frac{b}{2}-c\right)} \\
& \times \mathbf{M}_{3,5}^{1,3}\left[\left.\frac{t^{\alpha}}{\lambda_{2}^{\alpha}}\right|_{\left.(0,1),\left(1+\frac{b}{2}\right)\right),\left(1+\frac{b}{2}-c, 0\right),\left(1-\frac{b}{2}+c, 0\right),\left(\left(\frac{b}{2}-c\right)(1-\alpha)-d-p, \alpha\right)} ^{\left(1+\frac{b}{2}-c, 0\right),\left(1+\frac{b}{2}-c-d, 0\right),\left(1-\frac{b}{2}+c, 1\right)}\right] \\
+A H(t) p ! & \sum_{a=1}^{\infty} \frac{1}{a !}\left(\frac{-y}{\sqrt{v}}\right)^{a} \sum_{b=0}^{\infty}\left(\frac{-\theta}{\sqrt{v}}\right)^{b} \sum_{c=0}^{\infty} \frac{(-v K)^{c}}{c !} \sum_{d=0}^{\infty} \frac{(-H)^{d}}{d !}\left(\frac{1+\lambda_{1}}{\lambda_{2}^{\alpha}}\right)^{\left(\frac{a+b}{2}-c\right)} \\
\times & \mathbf{M}_{3,5}^{1,3}\left[\left.\frac{t^{\alpha}}{\lambda_{2}^{\alpha}}\right|_{(0,1),\left(1+\frac{a+b}{2}, 0\right),\left(1+\frac{a+b}{2}-c, 0\right),\left(1-\frac{a+b}{2}+c, 0\right),\left(\left(\frac{a+b}{2}-c\right)(1-\alpha)-d-p, \alpha\right)} ^{\left(1+\frac{a+b}{2}-c, 0\right),\left(1+\frac{a+b}{2}-c-d, 0\right),\left(1-\frac{a+b}{2}+c, 1\right)}\right],
\end{aligned}
$$

where the new generalized $\mathbf{M}$-function together property of the Fox H-function [30] is defined by

$$
\mathbf{M}_{k, n+1}^{1, k}\left[\left.z\right|_{(0,1),\left(1-b_{1}, B_{1}\right), \ldots\left(1-b_{n}, B_{n}\right)} ^{\left(1-a_{1}, A_{1}\right), \ldots\left(1-a_{k}, A_{k}\right)}\right]=t^{b_{n}-1} H_{k, n+1}^{1, k}\left[\left.z\right|_{(0,1),\left(1-b_{1}, B_{1}\right), \ldots\left(1-b_{n}, B_{n}\right)} ^{\left(1-a_{1}, A_{1}\right), \ldots\left(1-a_{k}, A_{k}\right)}\right]=t^{b_{n}-1} \sum_{p=0}^{\infty} \frac{(-z)^{p} \prod_{j=1}^{k} \Gamma\left(a_{j}+A_{j} p\right)}{p ! \prod_{j=1}^{n} \Gamma\left(b_{j}+B_{j} p\right)} .
$$




\subsection{Investigation of shear stress}

Using the Laplace transform technique, to Eq. (5), by using initial condition to Eq. (6) $)_{2}$, we get

$$
\bar{\tau}(y, q)=\frac{\mu}{\left(1+\lambda_{1}\right)}\left(1+\lambda_{2}^{\alpha} q^{\alpha}\right) \frac{\partial \bar{u}(y, q)}{\partial y},
$$

where $\bar{\tau}(y, q)$ is known as pre-image of $\tau(y, t)$. Now simplify Eq.(17) by using Eq. (12), we get

$$
\begin{gathered}
\bar{\tau}(y, q)=-\frac{A \mu p !}{\left(1+\lambda_{1}\right) \sqrt{v}} \frac{\left(1+\lambda_{2}^{\alpha} q^{\alpha}\right)}{q^{p+1}} \frac{1}{\left\{1+\theta\left[\frac{\left(1+\lambda_{1}\right)(q+H)+v K\left(1+\lambda_{2}^{\alpha} q^{\alpha}\right)}{v\left(1+\lambda_{2}^{\alpha} q^{\alpha}\right.}\right]^{\frac{1}{2}}\right\}} \\
\times\left[\frac{\left(1+\lambda_{1}\right)(q+H)+v K\left(1+\lambda_{2}^{\alpha} q^{\alpha}\right)}{v\left(1+\lambda_{2}^{\alpha} q^{\alpha}\right)}\right]^{\frac{1}{2}} \exp \left\{-\left[\frac{\left(1+\lambda_{1}\right)(q+H)+v K\left(1+\lambda_{2}^{\alpha} q^{\alpha}\right)}{v\left(1+\lambda_{2}^{\alpha} q^{\alpha}\right)}\right]^{\frac{1}{2}} y\right\} .
\end{gathered}
$$

Rewrite above equation in terms of series form, we obtain

$$
\begin{gathered}
\bar{\tau}(y, q)=-\frac{A \mu p !}{\sqrt{v}} \sum_{a=0}^{\infty} \frac{1}{a !}\left(\frac{-y}{\sqrt{v}}\right)^{a} \sum_{b=0}^{\infty}\left(\frac{-\theta}{\sqrt{v}}\right)^{b} \sum_{c=0}^{\infty} \frac{(-v K)^{c}}{c !} \sum_{d=0}^{\infty} \frac{(-H)^{d}}{d !}\left(\frac{1+\lambda_{1}}{\lambda_{2}^{\alpha}}\right)^{\left(\frac{a+b-1}{2}-c\right)} \\
\times \sum_{e=0}^{\infty} \frac{\Gamma\left(-\frac{a+b+1}{2}+c\right) \Gamma\left(-\frac{a+b+1}{2}+c+d\right) \Gamma\left(\frac{a+b-1}{2}-c+e\right)}{e ! \Gamma\left(-\frac{a+b+1}{2}\right) \Gamma\left(-\frac{a+b+1}{2}+c\right) \Gamma\left(\frac{a+b-1}{2}-c\right)} \frac{\left(-\lambda_{2}^{\alpha}\right)^{-e}}{q^{-\left(\frac{a+b}{2}-c\right)(1-\alpha)-\frac{1}{2}(1+\alpha)+d+\alpha e+p+1}} .
\end{gathered}
$$

Applying discrete inverse Laplace transform to Eq. (19), we get results in terms of series

$$
\begin{gathered}
\tau(y, t)=-\frac{A H(t) \mu p !}{\sqrt{v}} \sum_{a=0}^{\infty} \frac{1}{a !}\left(\frac{-y}{\sqrt{v}}\right)^{a} \sum_{b=0}^{\infty}\left(\frac{-\theta}{\sqrt{v}}\right)^{b} \sum_{c=0}^{\infty} \frac{(-v K)^{c}}{c !} \sum_{d=0}^{\infty} \frac{(-H)^{d}}{d !}\left(\frac{1+\lambda_{1}}{\lambda_{2}^{\alpha}}\right)^{\left(\frac{a+b-1}{2}-c\right)} \\
\times t^{-\left(\frac{a+b}{2}-c\right)(1-\alpha)-\frac{1}{2}(1+\alpha)+d+p} \sum_{e=0}^{\infty} \frac{\Gamma\left(-\frac{a+b+1}{2}+c\right) \Gamma\left(-\frac{a+b+1}{2}+c+d\right) \Gamma\left(\frac{a+b-1}{2}-c+e\right)}{e ! \Gamma\left(-\frac{a+b+1}{2}\right) \Gamma\left(-\frac{a+b+1}{2}+c\right) \Gamma\left(\frac{a+b-1}{2}-c\right)} \\
\times \frac{\left(-\frac{t^{\alpha}}{\lambda_{2}^{\alpha}}\right)^{e}}{\Gamma\left(-\left(\frac{a+b}{2}-c\right)(1-\alpha)-\frac{1}{2}(1+\alpha)+d+\alpha e+p+1\right)} .
\end{gathered}
$$

Express Eq. (20) in the form of generalized $\mathbf{M}$-function, we get the required shear stress

$$
\begin{aligned}
& \tau(y, t)=-\frac{A H(t) \mu p !}{\sqrt{v}} \sum_{a=0}^{\infty} \frac{1}{a !}\left(\frac{-y}{\sqrt{v}}\right)^{a} \sum_{b=0}^{\infty}\left(\frac{-\theta}{\sqrt{v}}\right)^{b} \sum_{c=0}^{\infty} \frac{(-v K)^{c}}{c !} \sum_{d=0}^{\infty} \frac{(-H)^{d}}{d !}\left(\frac{1+\lambda_{1}}{\lambda_{2}^{\alpha}}\right)^{\left(\frac{a+b-1}{2}-c\right)} \\
& \times \mathbf{M}_{3,5}^{1,3}\left[\left.\frac{t^{\alpha}}{\lambda_{2}^{\alpha}}\right|_{(0,1),\left(1+\frac{a+b+1}{2}, 0\right),\left(1+\frac{a+b+1}{2}-c, 0\right),\left(1-\frac{a+b-1}{2}+c, 0\right),\left(\left(\frac{a+b}{2}-c\right)(1-\alpha)+\frac{1}{2}(1+\alpha)-d-p, \alpha\right)} ^{\left(1+\frac{a+b+1}{2}-c, 0\right),\left(1+\frac{a+b+1}{2}-c-d, 0\right),\left(1-\frac{a+b-1}{2}+c, 1\right)}\right] .
\end{aligned}
$$

\section{Special cases}

\subsection{MHD ordinary Jeffrey fluid}

Making $\alpha \longrightarrow 1$ into Eqs. (16) and (21), we obtain following velocity field and corresponding shear stress for ordinary Jeffrey fluid. 


\subsection{Fractionalized Jeffrey fluid in porous plate without MHD}

Making $H \longrightarrow 0$ into Eqs. (16) and (21), we obtain following velocity field

$$
\begin{aligned}
& u(y, t)=A H(t) t^{p}+A H(t) p ! \sum_{b=1}^{\infty}\left(\frac{-\theta}{\sqrt{v}}\right)^{b} \sum_{c=0}^{\infty} \frac{(-v K)^{c}}{c !}\left(\frac{1+\lambda_{1}}{\lambda_{2}^{\alpha}}\right)^{\left(\frac{b}{2}-c\right)} \mathbf{M}_{2,4}^{1,2}\left[\left.\frac{t^{\alpha}}{\lambda_{2}^{\alpha}}\right|_{(0,1),\left(1+\frac{b}{2}, 0\right),\left(1-\frac{b}{2}+c, 0\right),\left(\left(\frac{b}{2}-c\right)(1-\alpha)-p, \alpha\right)} ^{\left(1+\frac{b}{2}-c, 0\right),\left(1-\frac{b}{2}+c, 1\right)}\right] \\
&+A H(t) p ! \sum_{a=1}^{\infty} \frac{1}{a !}\left(\frac{-y}{\sqrt{v}}\right)^{a} \sum_{b=0}^{\infty}\left(\frac{-\theta}{\sqrt{v}}\right)^{b} \sum_{c=0}^{\infty} \frac{(-v K)^{c}}{c !}\left(\frac{1+\lambda_{1}}{\lambda_{2}^{\alpha}}\right)^{\left(\frac{b}{2}-c\right)} \\
& \times \mathbf{M}_{2,4}^{1,2}\left[\left.\frac{t^{\alpha}}{\lambda_{2}^{\alpha}}\right|_{(0,1),\left(1+\frac{a+b}{2}, 0\right),\left(1-\frac{a+b}{2}+c, 0\right),\left(\left(\frac{a+b}{2}-c\right)(1-\alpha)-p, \alpha\right)} ^{\left(1+\frac{a+b}{2}-c, 0\right),\left(1-\frac{a+b}{2}+c, 1\right)}\right],
\end{aligned}
$$

and corresponding shear stress

$$
\begin{aligned}
\tau(y, t)= & -\frac{A H(t) \mu p !}{\sqrt{v}} \sum_{a=0}^{\infty} \frac{1}{a !}\left(\frac{-y}{\sqrt{v}}\right)^{a} \sum_{b=0}^{\infty}\left(\frac{-\theta}{\sqrt{v}}\right)^{b} \sum_{c=0}^{\infty} \frac{(-v K)^{c}}{c !}\left(\frac{1+\lambda_{1}}{\lambda_{2}^{\alpha}}\right)^{\left(\frac{a+b-1}{2}-c\right)} \\
& \times \mathbf{M}_{2,4}^{1,2}\left[\left.\frac{t^{\alpha}}{\lambda_{2}^{\alpha}}\right|_{(0,1),\left(1+\frac{a+b+1}{2}, 0\right),\left(1-\frac{a+b-1}{2}+c, 0\right),\left(\left(\frac{a+b}{2}-c\right)(1-\alpha)+\frac{1}{2}(1+\alpha)-p, \alpha\right)} ^{\left(1+\frac{a+b+1}{2}-c, 0\right),\left(1-\frac{a+b-1}{2}+c, 1\right)}\right] .
\end{aligned}
$$

\subsection{MHD fractionalized Jeffrey fluid without porous effects}

Making $K \longrightarrow 0$ into Eqs. (16) and (21), we obtain following velocity field

$$
\begin{gathered}
u(y, t)=A H(t) t^{p}+A H(t) p ! \sum_{b=1}^{\infty}\left(\frac{-\theta}{\sqrt{v}}\right)^{b} \sum_{d=0}^{\infty} \frac{(-H)^{d}}{d !}\left(\frac{1+\lambda_{1}}{\lambda_{2}^{\alpha}}\right)^{\frac{b}{2}} \mathbf{M}_{2,4}^{1,2}\left[\left.\frac{t^{\alpha}}{\lambda_{2}^{\alpha}}\right|_{(0,1),\left(1+\frac{b}{2}, 0\right),\left(1-\frac{b}{2}, 0\right),\left(\frac{b}{2}(1-\alpha)-d-p, \alpha\right)} ^{\left(1+\frac{b}{2}-0\right),\left(1-\frac{b}{2}, 1\right)}\right] \\
+A H(t) p ! \sum_{a=1}^{\infty} \frac{1}{a !}\left(\frac{-y}{\sqrt{v}}\right)^{a} \sum_{b=0}^{\infty}\left(\frac{-\theta}{\sqrt{v}}\right)^{b} \sum_{d=0}^{\infty} \frac{(-H)^{d}}{d !}\left(\frac{1+\lambda_{1}}{\lambda_{2}^{\alpha}}\right)^{\frac{a+b}{2}} \mathbf{M}_{2,4}^{1,2}\left[\left.\frac{t^{\alpha}}{\lambda_{2}^{\alpha}}\right|_{(0,1),\left(1+\frac{a+b}{2}, 0\right),\left(1-\frac{a+b}{2}, 0\right),\left(\frac{a+b}{2}(1-\alpha)-d-p, \alpha\right)} ^{\left(1+\frac{a+b}{2}-d, 0\right),\left(1-\frac{a+b}{2}, 1\right)}\right],
\end{gathered}
$$

and corresponding shear stress

$$
\begin{aligned}
\tau(y, t)= & -\frac{A H(t) \mu p !}{\sqrt{v}} \sum_{a=0}^{\infty} \frac{1}{a !}\left(\frac{-y}{\sqrt{v}}\right)^{a} \sum_{b=0}^{\infty}\left(\frac{-\theta}{\sqrt{v}}\right)^{b} \sum_{d=0}^{\infty} \frac{(-H)^{d}}{d !}\left(\frac{1+\lambda_{1}}{\lambda_{2}^{\alpha}}\right)^{\frac{a+b-1}{2}} \\
& \times \mathbf{M}_{2,4}^{1,2}\left[\left.\frac{t^{\alpha}}{\lambda_{2}^{\alpha}}\right|_{\left.(0,1),\left(1+\frac{a+b+1}{2}, 0\right),\left(1-\frac{a+b-1}{2}, 0\right),\left(\frac{a+b}{2}\right)(1-\alpha)+\frac{1}{2}(1-\alpha)-d-p, \alpha\right)} ^{\left(1+\frac{a+1}{2}-d, 0\right),\left(1-\frac{a+b-1}{2}, 1\right)}\right],
\end{aligned}
$$

for the above mentioned case.

\subsection{Fractionalized Jeffrey fluid without MHD and without porous effects}

Putting $K, H \longrightarrow 0$ into Eqs. (16) and (21) or using $K \longrightarrow 0$ into Eqs. (22) and (23) or making $H \longrightarrow 0$ into Eqs. (24) and (25), we get following velocity field

$$
u(y, t)=A H(t) t^{p}+A H(t) p ! \sum_{b=1}^{\infty}\left(\frac{-\theta}{\sqrt{v}}\right)^{b}\left(\frac{1+\lambda_{1}}{\lambda_{2}^{\alpha}}\right)^{\frac{b}{2}} \mathbf{M}_{1,3}^{1,1}\left[\left.\frac{t^{\alpha}}{\lambda_{2}^{\alpha}}\right|_{(0,1),\left(1-\frac{b}{2}, 0\right),\left(\frac{b}{2}(1-\alpha)-p, \alpha\right)} ^{\left(1-\frac{b}{2}, 1\right)}\right]
$$




$$
+A H(t) p ! \sum_{a=1}^{\infty} \frac{1}{a !}\left(\frac{-y}{\sqrt{v}}\right)^{a} \sum_{b=0}^{\infty}\left(\frac{-\theta}{\sqrt{v}}\right)^{b}\left(\frac{1+\lambda_{1}}{\lambda_{2}^{\alpha}}\right)^{\frac{a+b}{2}} \mathbf{M}_{1,3}^{1,1}\left[\left.\frac{t^{\alpha}}{\lambda_{2}^{\alpha}}\right|_{(0,1),\left(1-\frac{a+b}{2}, 0\right),\left(\frac{a+b}{2}(1-\alpha)-p, \alpha\right)} ^{\left(1-\frac{a+b}{2}, 1\right)}\right],
$$

and

$$
\begin{aligned}
\tau(y, t)= & -\frac{A H(t) \mu p !}{\sqrt{v}} \sum_{a=0}^{\infty} \frac{1}{a !}\left(\frac{-y}{\sqrt{v}}\right)^{a} \sum_{b=0}^{\infty}\left(\frac{-\theta}{\sqrt{v}}\right)^{b}\left(\frac{1+\lambda_{1}}{\lambda_{2}^{\alpha}}\right)^{\frac{a+b-1}{2}} \\
& \times \mathbf{M}_{1,3}^{1,1}\left[\left.\frac{t^{\alpha}}{\lambda_{2}^{\alpha}}\right|_{(0,1),\left(1-\frac{a+b-1}{2}, 0\right),\left(\frac{a+b}{2}(1-\alpha)+\frac{1}{2}(1+\alpha)-p, \alpha\right)} ^{\left(1-\frac{a+b-1}{2}, 1\right)}\right.
\end{aligned}
$$

corresponding shear stress fractionalized Jeffrey fluid without MHD and without porus effects.

\subsection{MHD fractionalized second grade fluid on porous plate}

Letting $\lambda_{1} \longrightarrow 0$ into Eqs. (16) and (21), we obtain the equivalent solutions for velocity field and shear stress for MHD fractionalized second grade fluid, which are

$$
\begin{aligned}
& u(y, t)=A H(t) t^{p}+A H(t) p ! \sum_{b=1}^{\infty}\left(\frac{-\theta}{\sqrt{v}}\right)^{b} \sum_{c=0}^{\infty} \frac{(-v K)^{c}}{c !} \sum_{d=0}^{\infty} \frac{(-H)^{d}}{d !}\left(\lambda_{2}^{\alpha}\right)^{-\left(\frac{b}{2}-c\right)} \\
& \times \mathbf{M}_{3,5}^{1,3}\left[\left.\frac{t^{\alpha}}{\lambda_{2}^{\alpha}}\right|_{(0,1),\left(1+\frac{b}{2}, 0\right),\left(1+\frac{b}{2}-c, 0\right),\left(1-\frac{b}{2}+c, 0\right),\left(\left(\frac{b}{2}-c\right)(1-\alpha)-d-p, \alpha\right)} ^{\left(1+\frac{b}{2}-c, 0\right),\left(1+\frac{b}{2}-c-d, 0\right),\left(1-\frac{b}{2}+c, 1\right)}\right] \\
& +A H(t) p ! \sum_{a=1}^{\infty} \frac{1}{a !}\left(\frac{-y}{\sqrt{v}}\right)^{a} \sum_{b=0}^{\infty}\left(\frac{-\theta}{\sqrt{v}}\right)^{b} \sum_{c=0}^{\infty} \frac{(-v K)^{c}}{c !} \sum_{d=0}^{\infty} \frac{(-H)^{d}}{d !}\left(\lambda_{2}^{\alpha}\right)^{-\left(\frac{a+b}{2}-c\right)} \\
& \times \mathbf{M}_{3,5}^{1,3}\left[\left.\frac{t^{\alpha}}{\lambda_{2}^{\alpha}}\right|_{(0,1),\left(1+\frac{a+b}{2}, 0\right),\left(1+\frac{a+b}{2}, 0\right),\left(1-\frac{a+b}{2}+c, 0\right),\left(\left(\frac{a+b}{2}-c\right)(1-\alpha)-d-p, \alpha\right)} ^{\left(1+\frac{a+b}{2}-c, 0\right),\left(1+\frac{a+b}{2}-c-d, 0\right),\left(1-\frac{a+b}{2}+c, 1\right)}\right], \\
& \tau(y, t)=-\frac{A H(t) \mu p !}{\sqrt{v}} \sum_{a=0}^{\infty} \frac{1}{a !}\left(\frac{-y}{\sqrt{v}}\right)^{a} \sum_{b=0}^{\infty}\left(\frac{-\theta}{\sqrt{v}}\right)^{b} \sum_{c=0}^{\infty} \frac{(-v K)^{c}}{c !} \sum_{d=0}^{\infty} \frac{(-H)^{d}}{d !}\left(\lambda_{2}^{\alpha}\right)^{-\left(\frac{a+b-1}{2}-c\right)} \\
& \times \mathbf{M}_{3,5}^{1,3}\left[\left.\frac{t^{\alpha}}{\lambda_{2}^{\alpha}}\right|_{(0,1),\left(1+\frac{a+b+1}{2}, 0\right),\left(1+\frac{a+b+1}{2}-c, 0\right),\left(1-\frac{a+b-1}{2}+c, 0\right),\left(\left(\frac{a+b}{2}-c\right)(1-\alpha)+\frac{1}{2}(1+\alpha)-d-p, \alpha\right)} ^{\left(1+\frac{a+1}{2}-c, 0\right),\left(1+\frac{a+b+1}{2}-c-d, 0\right),\left(1-\frac{a+b-1}{2}+c, 1\right)}\right] .
\end{aligned}
$$

\subsection{MHD fractionalized Jeffrey fluid on porous plate for $\lambda_{2} \longrightarrow 0$}

Using $\lambda_{2} \longrightarrow 0$ into Eqs. (16) and (21), we get

$$
\begin{aligned}
u(y, t)=A H(t) t^{p}+ & A H(t) p ! \sum_{b=1}^{\infty}\left(\frac{-\theta}{\sqrt{v}}\right)^{b} \sum_{c=0}^{\infty} \frac{(-v K)^{c}}{c !}\left(1+\lambda_{1}\right)^{\left(\frac{b}{2}-c\right)} \mathbf{M}_{2,4}^{1,2}\left[\left.(H t)\right|_{(0,1),\left(1+\frac{b}{2}, 0\right),\left(1-\frac{b}{2}+c, 0\right),\left(\frac{b}{2}-c-p, 1\right)} ^{\left(1+\frac{b}{2}-c, 0\right),\left(1-\frac{b}{2}+c, 1\right)}\right] \\
& +A H(t) p ! \sum_{a=1}^{\infty} \frac{1}{a !}\left(\frac{-y}{\sqrt{v}}\right)^{a} \sum_{b=0}^{\infty}\left(\frac{-\theta}{\sqrt{v}}\right)^{b} \sum_{c=0}^{\infty} \frac{(-v K)^{c}}{c !}\left(1+\lambda_{1}\right)^{\left(\frac{a+b}{2}-c\right)}
\end{aligned}
$$




$$
\begin{gathered}
\times \mathbf{M}_{2,4}^{1,2}\left[\left.(H t)\right|_{(0,1),\left(1+\frac{a+b}{2}, 0\right),\left(1-\frac{a+b}{2}+c, 0\right),\left(\frac{a+b}{2}-c-p, 1\right)} ^{\left(1+\frac{a+b}{2}-c, 0\right),\left(1-\frac{a+b}{2}+c, 1\right)}\right] \\
\tau(y, t)=-\frac{A H(t) \mu p !}{\sqrt{v}} \sum_{a=0}^{\infty} \frac{1}{a !}\left(\frac{-y}{\sqrt{v}}\right)^{a} \sum_{b=0}^{\infty}\left(\frac{-\theta}{\sqrt{v}}\right)^{b} \sum_{c=0}^{\infty} \frac{(-v K)^{c}}{c !}\left(1+\lambda_{1}\right)^{\left(\frac{a+b-1}{2}-c\right)} \\
\times \mathbf{M}_{2,4}^{1,2}\left[\left.(H t)\right|_{(0,1),\left(1+\frac{a+b+1}{2}, 0\right),\left(1-\frac{a+b-1}{2}+c, 0\right),\left(\frac{a+b+1}{2}-c-p, 1\right)} ^{\left(1+\frac{a+b+1}{2}-c, 0\right),\left(1-\frac{a+b-1}{2}+c, 1\right)}\right] .
\end{gathered}
$$

\subsection{MHD Newtonian fluid on porous plate}

Putting $\lambda_{1}, \lambda_{2} \longrightarrow 0$ into Eqs. (16) and (21) or using $\lambda_{2} \longrightarrow 0$ into Eqs. (28) and (29) or making $\lambda_{1} \longrightarrow 0$ into Eqs. (30) and (31), we get following velocity field

$$
\begin{gathered}
u(y, t)=A H(t) t^{p}+A H(t) p ! \sum_{b=1}^{\infty}\left(\frac{-\theta}{\sqrt{v}}\right)^{b} \sum_{c=0}^{\infty} \frac{(-v K)^{c}}{c !} \mathbf{M}_{2,4}^{1,2}\left[\left.(H t)\right|_{(0,1),\left(1+\frac{b}{2}, 0\right),\left(1-\frac{b}{2}+c, 0\right),\left(\frac{b}{2}-c-p, 1\right)} ^{\left(1+\frac{b}{2}-c, 0\right),\left(1-\frac{b}{2}+c, 1\right)}\right] \\
+A H(t) p ! \sum_{a=1}^{\infty} \frac{1}{a !}\left(\frac{-y}{\sqrt{v}}\right)^{a} \sum_{b=0}^{\infty}\left(\frac{-\theta}{\sqrt{v}}\right)^{b} \sum_{c=0}^{\infty} \frac{(-v K)^{c}}{c !} \mathbf{M}_{2,4}^{1,2}\left[\left.(H t)\right|_{(0,1),\left(1+\frac{a+b}{2}, 0\right),\left(1-\frac{a+b}{2}+c, 0\right),\left(\frac{a+b}{2}-c-p, 1\right)} ^{\left(1+\frac{a+b}{2}-c, 0\right),\left(1-\frac{a+b}{2}+c, 1\right)}\right],
\end{gathered}
$$

and corresponding shear stress

$$
\begin{aligned}
\tau(y, q) & =-\frac{A H(t) \mu p !}{\sqrt{v}} \sum_{a=0}^{\infty} \frac{1}{a !}\left(\frac{-y}{\sqrt{v}}\right)^{a} \sum_{b=0}^{\infty}\left(\frac{-\theta}{\sqrt{v}}\right)^{b} \sum_{c=0}^{\infty} \frac{(-v K)^{c}}{c !} \\
& \times \mathbf{M}_{2,4}^{1,2}\left[\left.H t\right|_{(0,1),\left(1+\frac{a+b+1}{2}, 0\right),\left(1-\frac{a+b-1}{2}+c, 0\right),\left(\frac{a+b+1}{2}-c-p, 1\right)} ^{\left(1+\frac{a+b+1}{2}-c, 0\right),\left(1-\frac{a+b-1}{2}+c, 1\right)}\right] .
\end{aligned}
$$

for MHD Newtonian fluid on porous plate.

\subsection{MHD Newtonian fluid without porous effect}

Making $K \longrightarrow 0$ into Eqs. (32) and (33), we obtain following velocity field

$$
\begin{aligned}
& \left.u(y, t)=A H(t) t^{p}+\left.A H(t) p ! \sum_{b=1}^{\infty}\left(\frac{-\theta}{\sqrt{v}}\right)^{b} \mathbf{M}_{1,3}^{1,1}[H t)\right|_{(0,1),\left(1-\frac{b}{2}, 0\right),\left(\frac{b}{2}-p, 1\right)} ^{\left(1-\frac{b}{2}, 1\right)}\right] \\
& +A H(t) p ! \sum_{a=1}^{\infty} \frac{1}{a !}\left(\frac{-y}{\sqrt{v}}\right)^{a} \sum_{b=0}^{\infty}\left(\frac{-\theta}{\sqrt{v}}\right)^{b} \mathbf{M}_{1,3}^{1,1}\left[\left.(H t)\right|_{(0,1),\left(1-\frac{a+b}{2}, 0\right),\left(\frac{a+b}{2}-p, 1\right)} ^{\left(1-\frac{a+b}{2}, 1\right)}\right]
\end{aligned}
$$

and corresponding shear stress

$$
\tau(y, t)=-\frac{A H(t) \mu p !}{\sqrt{v}} \sum_{a=0}^{\infty} \frac{1}{a !}\left(\frac{-y}{\sqrt{v}}\right)^{a} \sum_{b=0}^{\infty}\left(\frac{-\theta}{\sqrt{v}}\right)^{b} \mathbf{M}_{1,3}^{1,1}\left[\left.(H t)\right|_{(0,1),\left(1-\frac{a+b-1}{2}, 0\right),\left(\frac{a+b+1}{2}-p, 1\right)} ^{\left(1-\frac{a+b-1}{2}, 1\right)}\right] .
$$




\subsection{MHD fractionalized Jeffrey fluid without slip effects on porous plate}

Letting $\theta \longrightarrow 0$ into Eqs. (16) and (21), we get following velocity field

$$
\begin{aligned}
u(y, t)= & A H(t) t^{p}+A H(t) p ! \sum_{a=1}^{\infty} \frac{1}{a !}\left(\frac{-y}{\sqrt{v}}\right)^{a} \sum_{c=0}^{\infty} \frac{(-v K)^{c}}{c !} \sum_{d=0}^{\infty} \frac{(-H)^{d}}{d !}\left(\frac{1+\lambda_{1}}{\lambda_{2}^{\alpha}}\right)^{\frac{a}{2}-c} \\
& \times \mathbf{M}_{3,5}^{1,3}\left[\left.\frac{t^{\alpha}}{\lambda_{2}^{\alpha}}\right|_{(0,1),\left(1+\frac{a}{2}, 0\right)\left(1+\frac{a}{2}-c, 0\right),\left(1-\frac{a}{2}+c, 0\right),\left(\left(\frac{a}{2}-c\right)(1-\alpha)-d-p, \alpha\right)} ^{\left(1+\frac{a}{2}-c, 0\right),\left(1+\frac{a}{2}-c-d, 0\right),\left(1-\frac{a}{2}+c, 1\right)}\right],
\end{aligned}
$$

and corresponding shear stress for mentioned case

$$
\begin{gathered}
\tau(y, t)=-\frac{A H(t) \mu p !}{\sqrt{v}} \sum_{a=0}^{\infty} \frac{1}{a !}\left(\frac{-y}{\sqrt{v}}\right)^{a} \sum_{c=0}^{\infty} \frac{(-v k)^{c}}{c !} \sum_{d=0}^{\infty} \frac{(-H)^{d}}{d !}\left(\frac{1+\lambda_{1}}{\lambda_{2}^{\alpha}}\right)^{\frac{a-1}{2}-c} \\
\times \mathbf{M}_{3,5}^{1,3}\left[\left.\frac{t^{\alpha}}{\lambda_{2}^{\alpha}}\right|_{(0,1),\left(1+\frac{a+1}{2}, 0\right)\left(1+\frac{a+1}{2}-c, 0\right),\left(1-\frac{a-1}{2}+c, 0\right),\left(\left(\frac{a}{2}-c\right)(1-\alpha)+\frac{1}{2}(1+\alpha)-d-p, \alpha\right)} ^{(1+c, 0),\left(1+\frac{a+1}{2}-c-d, 0\right),\left(1-\frac{a-1}{2}+c, 1\right)}\right] .
\end{gathered}
$$

\subsection{Fractionalized Jeffrey fluid without slip \& MHD effects on porous plate}

Making $H \longrightarrow 0$ into Eqs. (36) and (37), we obtain the required solutions

$$
\begin{gathered}
u(y, t)=A H(t) t^{p}+A H(t) p ! \sum_{a=1}^{\infty} \frac{1}{a !}\left(\frac{-y}{\sqrt{v}}\right)^{a} \sum_{c=0}^{\infty} \frac{(-v K)^{c}}{c !}\left(\frac{1+\lambda_{1}}{\lambda_{2}^{\alpha}}\right)^{\frac{a}{2}-c} \\
\times \mathbf{M}_{2,4}^{1,2}\left[\left.\frac{t^{\alpha}}{\lambda_{2}^{\alpha}}\right|_{(0,1),\left(1+\frac{a}{2}, 0\right),\left(1-\frac{a}{2}+c, 0\right),\left(\left(\frac{a}{2}-c\right)(1-\alpha)-p, \alpha\right)} ^{\left(1+\frac{a}{2}-c, 0\right),\left(1-\frac{a}{2}+c, 1\right)}\right], \\
\tau(y, t)=-\frac{A H(t) \mu p !}{\sqrt{v}} \sum_{a=0}^{\infty} \frac{1}{a !}\left(\frac{-y}{\sqrt{v}}\right)^{a} \sum_{c=0}^{\infty} \frac{(-v K)^{c}}{c !}\left(\frac{1+\lambda_{1}}{\lambda_{2}^{\alpha}}\right)^{\frac{a-1}{2}-c} \\
\times \mathbf{M}_{2,4}^{1,2}\left[\left.\frac{t^{\alpha}}{\lambda_{2}^{\alpha}}\right|_{(0,1),\left(1+\frac{a+1}{2}, 0\right),\left(1-\frac{a-1}{2}+c, 0\right),\left(\left(\frac{a+1}{2}-c\right)(1-\alpha)+\frac{1}{2}(1+\alpha)-p, \alpha\right)} ^{\left(1+\frac{a+1}{2}-c, 0\right),\left(1-\frac{a-1}{2}+c, 1\right)}\right] .
\end{gathered}
$$

\subsection{MHD fractionalized Jeffrey fluid without slip \& porous effects}

Putting $K \longrightarrow 0$, into the Eqs. (36) and (37), we obtain the following velocity field and the shear stress

$$
\begin{gathered}
u(y, t)=A H(t) t^{p}+A H(t) p ! \sum_{a=1}^{\infty} \frac{1}{a !}\left(\frac{-y}{\sqrt{v}}\right)^{a} \sum_{d=0}^{\infty} \frac{(-H)^{d}}{d !}\left(\frac{1+\lambda_{1}}{\lambda_{2}^{\alpha}}\right)^{\frac{a}{2}} \\
\times \mathbf{M}_{2,4}^{1,2}\left[\left.\frac{t^{\alpha}}{\lambda_{2}^{\alpha}}\right|_{(0,1),\left(1+\frac{a}{2}, 0\right),\left(1-\frac{a}{2}, 0\right),\left(\frac{a}{2}(1-\alpha)-d-p, \alpha\right)} ^{\left(1+\frac{a}{2}-d, 0\right),\left(1-\frac{a}{2}, 1\right)}\right], \\
\tau(y, t)=-\frac{A H(t) \mu p !}{\sqrt{v}} \sum_{a=0}^{\infty} \frac{1}{a !}\left(\frac{-y}{\sqrt{v}}\right)^{a} \sum_{d=0}^{\infty} \frac{(-H)^{d}}{d !}\left(\frac{1+\lambda_{1}}{\lambda_{2}^{\alpha}}\right)^{\frac{a-1}{2}} \\
\times \mathbf{M}_{2,4}^{1,2}\left[\left.\frac{t^{\alpha}}{\lambda_{2}^{\alpha}}\right|_{(0,1),\left(1+\frac{a+1}{2}, 0\right),\left(1-\frac{a-1}{2}, 0\right),\left(\frac{a+1}{2}(1-\alpha)+\frac{1}{2}(1+\alpha)-d-p, \alpha\right)} ^{\left(1+\frac{a+1}{2}-d, 0\right),\left(1-\frac{a-1}{2}, 1\right)}\right] .
\end{gathered}
$$




\subsection{Fractionalized Jeffrey fluid without slip, MHD \& porous effects}

Making $H \longrightarrow 0$ into the Eqs. (40) and (41), we obtain following velocity field

$$
u(y, t)=A H(t) t^{p}+A H(t) p ! \sum_{a=1}^{\infty} \frac{1}{a !}\left(\frac{-y}{\sqrt{v}}\right)^{a}\left(\frac{1+\lambda_{1}}{\lambda_{2}^{\alpha}}\right)^{\frac{a}{2}} \mathbf{M}_{1,3}^{1,1}\left[\left.\frac{t^{\alpha}}{\lambda_{2}^{\alpha}}\right|_{(0,1),\left(1-\frac{a}{2}, 0\right),\left(\frac{a}{2}(1-\alpha)-p, \alpha\right)} ^{\left(1-\frac{\alpha}{2}, 1\right)}\right],
$$

and corresponding shear stress

$$
\tau(y, t)=-\frac{A H(t) \mu p !}{\sqrt{v}} \sum_{a=0}^{\infty} \frac{1}{a !}\left(\frac{-y}{\sqrt{v}}\right)^{a}\left(\frac{1+\lambda_{1}}{\lambda_{2}^{\alpha}}\right)^{\frac{a-1}{2}} \mathbf{M}_{1,3}^{1,1}\left[\left.\frac{t^{\alpha}}{\lambda_{2}^{\alpha}}\right|_{(0,1),\left(1-\frac{a-1}{2}, 0\right),\left(\frac{a}{2}(1-\alpha)+\frac{1}{2}(1+\alpha)-p, \alpha\right)} ^{\left(1-\frac{a-1}{2}, 1\right)}\right] .
$$

\subsection{MHD fractionalized second grade fluid without slip effect on porous plate}

Using $\lambda_{1} \longrightarrow 0$ into Eqs. (36) and (37), we obtain the equivalent solution for velocity and shear stress for second grade fluid without slip effect

$$
\begin{gathered}
u(y, t)=A H(t) t^{p}+A H(t) p ! \sum_{a=1}^{\infty} \frac{1}{a !}\left(\frac{-y}{\sqrt{v}}\right)^{a} \sum_{c=0}^{\infty} \frac{(-v K)^{c}}{c !} \sum_{d=0}^{\infty} \frac{(-H)^{d}}{d !}\left(\lambda_{2}^{\alpha}\right)^{-\left(\frac{a}{2}-c\right)} \\
\times \mathbf{M}_{3,5}^{1,3}\left[\left.\frac{t^{\alpha}}{\lambda_{2}^{\alpha}}\right|_{(0,1),\left(1+\frac{a}{2}, 0\right)\left(1+\frac{a}{2}-c, 0\right),\left(1-\frac{a}{2}+c, 0\right),\left(\left(\frac{a}{2}-c\right)(1-\alpha)-d-p, \alpha\right)} ^{\left(1+\frac{a}{2}-c, 0\right),\left(1+\frac{a}{2}-c-d, 0\right),\left(1-\frac{a}{2}+c, 1\right)}\right] \\
\tau(y, t)=-\frac{A H(t) \mu p !}{\sqrt{v}} \sum_{a=0}^{\infty} \frac{1}{a !}\left(\frac{-y}{\sqrt{v}}\right)^{a} \sum_{c=0}^{\infty} \frac{(-v K)^{c}}{c !} \sum_{d=0}^{\infty} \frac{(-H)^{d}}{d !}\left(\lambda_{2}^{\alpha}\right)^{-\frac{a-1}{2}-c} \\
\times \mathbf{M}_{3,5}^{1,3}\left[\left.\frac{t^{\alpha}}{\lambda_{2}^{\alpha}}\right|_{(0,1),\left(1+\frac{a+1}{2}, 0\right)\left(1+\frac{a+1}{2}-c, 0\right),\left(1-\frac{a-1}{2}+c, 0\right),\left(\left(\frac{a+1}{2}-c\right)(1-\alpha)+\frac{1}{2}(1+\alpha)-d-p, \alpha\right)} ^{\left(1+\frac{a+1}{2}-c, 0\right),\left(1+\frac{a+1}{2}-c-d, 0\right),\left(1-\frac{a-1}{2}+c, 1\right)}\right] .
\end{gathered}
$$

\subsection{MHD fractionalized Jeffrey fluid without slip effect on porous plate for $\lambda_{2} \longrightarrow 0$}

Assuming $\lambda_{2} \longrightarrow 0$ into the Eqs. (36) and (37), we obtain following velocity field

$$
\begin{aligned}
u(y, t)= & A H(t) t^{p}+A H(t) p ! \sum_{a=1}^{\infty} \frac{1}{a !}\left(\frac{-y}{\sqrt{v}}\right)^{a} \sum_{c=0}^{\infty} \frac{(-v K)^{c}}{c !}\left(1+\lambda_{1}\right)^{\left(\frac{a}{2}-c\right)} \\
& \times \mathbf{M}_{2,4}^{1,2}\left[\left.(H t)\right|_{(0,1),\left(1+\frac{a}{2}, 0\right),\left(1-\frac{a}{2}+c, 0\right),\left(\frac{a}{2}-c-p, 1\right)} ^{\left(1+\frac{a}{2}-c, 0\right),\left(1-\frac{a}{2}+c, 1\right)}\right],
\end{aligned}
$$

and corresponding shear stress

$$
\begin{aligned}
\tau(y, t)= & -\frac{A H(t) \mu p !}{\sqrt{v}} \sum_{a=0}^{\infty} \frac{1}{a !}\left(\frac{-y}{\sqrt{v}}\right)^{a} \sum_{c=0}^{\infty} \frac{(-v K)^{c}}{c !}\left(1+\lambda_{1}\right)^{\left(\frac{a-1}{2}-c\right)} \\
& \times \mathbf{M}_{2,4}^{1,2}\left[\left.H t\right|_{(0,1),\left(1+\frac{a+1}{2}, 0\right),\left(1-\frac{a-1}{2}+c, 0\right),\left(\frac{a+1}{2}-c-p, 1\right)} ^{\left(1+\frac{a+1}{2}-c, 0\right),\left(1-\frac{a-1}{2}+c, 1\right)}\right] .
\end{aligned}
$$

corresponding to the above case. 


\subsection{MHD Newtonian fluid without slip effect on porous plate}

Making $\lambda_{1} \longrightarrow 0$ into Eqs. (46) and (47), we get solutions for MHD Newtonian fluid without slip effect on porous plate

$$
\begin{gathered}
u(y, t)=A H(t) t^{p}+A H(t) p ! \sum_{a=1}^{\infty} \frac{1}{a !}\left(\frac{-y}{\sqrt{v}}\right)^{a} \sum_{c=0}^{\infty} \frac{(-v K)^{c}}{c !} \mathbf{M}_{2,4}^{1,2}\left[\left.H t\right|_{(0,1),\left(1+\frac{a}{2}, 0\right),\left(1-\frac{a}{2}+c, 0\right),\left(\frac{a}{2}-c-p, 1\right)} ^{\left(1+\frac{a}{2}-c, 0\right),\left(1-\frac{a}{2}+c, 1\right)}\right], \\
\tau(y, t)=-\frac{A H(t) \mu p !}{\sqrt{v}} \sum_{a=0}^{\infty} \frac{1}{a !}\left(\frac{-y}{\sqrt{v}}\right)^{a} \sum_{c=0}^{\infty} \frac{(-v K)^{c}}{c !} \mathbf{M}_{2,4}^{1,2}\left[\left.H t\right|_{(0,1),\left(1+\frac{a+1}{2}, 0\right),\left(1-\frac{a-1}{2}+c, 0\right),\left(\frac{a+1}{2}-c-p, 1\right)} ^{\left(1+\frac{a+1}{2}-c, 0\right),\left(1-\frac{a-1}{2}+c, 1\right)}\right] .
\end{gathered}
$$

\subsection{MHD Newtonian fluid without slip \& porous effects}

Making $K \longrightarrow 0$, in Eq. (48) and (49), we get the required solutions for mentioned case as

$$
\begin{gathered}
u(y, t)=A H(t) t^{p}+A H(t) p ! \sum_{a=1}^{\infty} \frac{1}{a !}\left(\frac{-y}{\sqrt{v}}\right)^{a} \mathbf{M}_{1,3}^{1,1}\left[\left.H t\right|_{(0,1),\left(1-\frac{a}{2}, 0\right),\left(\frac{a}{2}-p, 1\right)} ^{\left(1-\frac{a}{2}, 1\right)}\right], \\
\tau(y, t)=-\frac{A H(t) \mu p !}{\sqrt{v}} \sum_{a=0}^{\infty} \frac{1}{a !}\left(\frac{-y}{\sqrt{v}}\right)^{a} \mathbf{M}_{1,3}^{1,1}\left[\left.H t\right|_{(0,1),\left(1-\frac{a-1}{2}, 0\right),\left(\frac{a+1}{2}-p, 1\right)} ^{\left(1-\frac{a-1}{2}, 1\right)}\right] .
\end{gathered}
$$

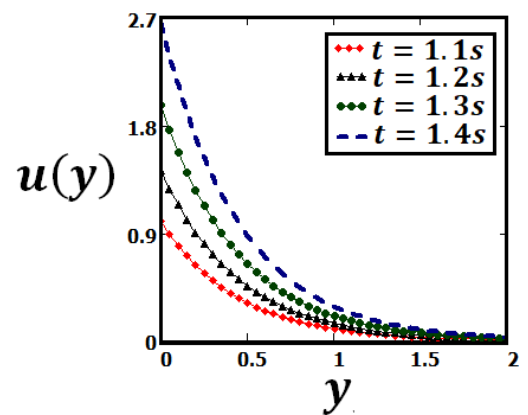

(a)

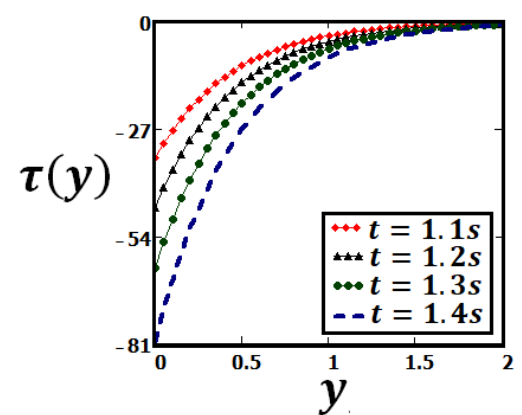

(b)

Figure 2: Profiles of the velocity field $u(y, t)$ and the shear stress $\tau(y, t)$ for fractionalized Jeffrey fluid given by Eqs. (16) and (21), for $A=$ $1, \quad v=1.427, \mu=19, \lambda_{1}=3, \lambda_{2}=2, H=0.5, K=2, \alpha=0.5, \theta=0.2, p=4$ and different values of $t$.

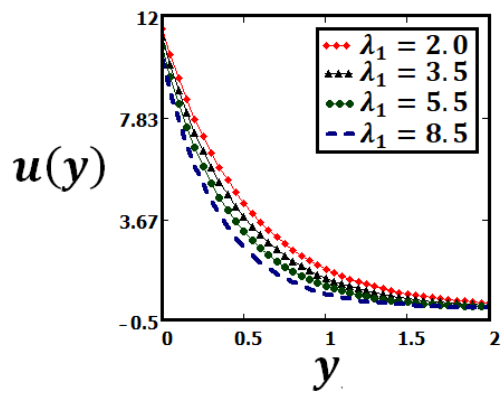

(a)

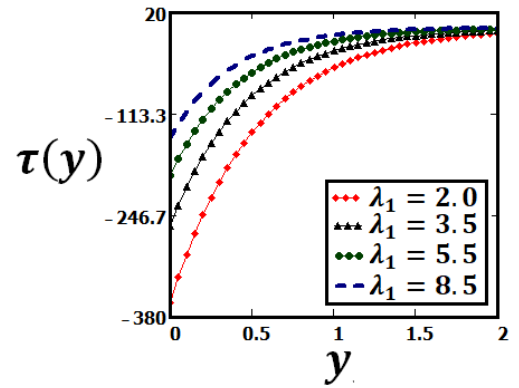

(b)

Figure 3: Profiles of the velocity field $u(y, t)$ and the shear stress $\tau(y, t)$ for fractionalized Jeffrey fluid given by Eqs. (16) and (21), for $A=$ $1, \quad v=1.427, \mu=19, \lambda_{2}=2, H=0.5, K=2, \alpha=0.5, \theta=0.2, p=4, t=2 s$ and different values of $\lambda_{1}$. 


\section{Numerical results and conclusions}

In this paper, we have used fractional calculus because it is proved that for practical purpose it is very useful in engineering and sciences, fractional approach is help full when it comes to generalization of complex dynamics of fluid motion. It shown in various field like electromagnetism, electrochemistry finance, signal process and biochemistry, the important application subject to magnetic field is the flow of electrical conducting fluid, MHD deals with the dynamics of electrically conducting fluids. In addition, the Magnetohydrodynamics (MHD) stream problem has increased significant premium in view of its extensive engineering and medical applications. The standards of MHD are utilized in the design of heat exchangers, pumps, flow meters, radar systems, power generation and so on. The significant of Jeffrey fluid is that the result is limited as for polymer industry is concerned.

The object of this paper is to determine the exact solutions unsteady flow of MHD fractionalized Jeffrey fluid over an accelerating porous plate under the consideration of first order slip boundary condition. The analytic solution is obtained for the velocity field and shear stress in terms of generalized $\mathbf{M}$-function and by employing Laplace transform techniques. At time $\mathrm{t}=0$ the fluid velocity field is zero, but at $t=0^{+}$the fluid is immediately moved with velocity $A t^{p}$ in its plate with assuming first order linear slip between fluid and the plate. Here the velocity distribution and shear stress of fluid are determined against the physical parameters, such as, MHD parameter $\mathrm{H}$, porosity $\mathrm{K}$, slip impact $\theta$, relaxation and retardation time parameters $\lambda_{1}$ and $\lambda_{2}$ and fractional parameter $\alpha$. The obtained results for velocity fluid flow and its corresponding shear stress are exhibited through limiting cases by taking $\alpha=1, H=0, K=0, M=0, \lambda_{1}=\lambda_{2}=0$ and $\theta=0$. Here the results of fractionalized MHD Jeffrey fluid are minimized into the ordinary Jeffrey fluid, without MHD effect, without porosity effect, Newtonian fluid and with and without slip effects. Furthermore, in order to discuss some physical aspect of the determined solutions, we have made graphs of velocity and shear stress against vertical height $y$. In all sketched diagram the values of physical parameters are taken as common, for instance, $A=1, v=1.427, \mu=19, \lambda_{1}=3, \lambda_{2}=2, H=$ $0.5, K=2, \alpha=0.5, \theta=0.2, p=4$.

The impacts of time are vital for us to discuss here, Figs. 2 is represented that the velocity distribution and shear stress at different values in $t$, it is noted from these diagram, the velocity field and shear stress (in absolute value) enhance by increase in $t$. Figs. 3 is sketched to show the behavior of the Jeffrey fluid parameter $\lambda_{1}$ on the flow of velocity field and shear stress, it is shown that velocity distribution and shear stress (in absolute value) is decreased with increased parameter $\lambda_{1}$. The impact of Jeffrey parameter $\lambda_{2}$ on velocity and shear stress is shown in Figs. 4, it is clearly seen in Figs. 4, that the velocity distribution and its corresponding shear stress increase with increases Jeffrey parameter $\lambda_{2}$. From sketched diagrams 3 and 4, we have observed both parameters $\lambda_{1}$ and $\lambda_{2}$ have opposite effects on fluid flows. It is clarified from sketched Figs. 5 and 6 that the effects of the magnetohydrodynamic(MHD) parameter $M$ and porosity parameter $K$ on velocity field and shear stress have similar. It is noticeable that velocity field is decreasing and shear stress is decreasing on large domain with increasing values of $M$ and $K$. Figs. 7 depicts the velocity field and shear stress at various values of kinematic viscosity $v$. It is clear velocity field as well as shear stress both increasing with increasing values of $v$. It is noted that the effects of the fractional parameter $\alpha$ on both velocity field and shear stress are increased by increasing $\alpha$ as shown in Figs. 8. In order to discuss the influence of slip parameter $\theta$, we have sketched Figs. 9, where it is observed that velocity field and shears tress are strong functions of slip parameter $\theta$, it presents that velocity field and shear stress both decreases with increases slip parameter $\theta$, as expected. Figs. 10 represents the velocity field and shear stress enhance by enhance values of the parameter $p$.

Finally, for analysis for the velocity field and shear stress to four different fluid models (fractionalized Jeffrey, ordinary Jeffrey, MHD Newtonian fluid with porosity, simple Newtonian fluids) for three different values of slip effect parameter $\theta=0.2,2.0$ and 0 . These fluid models are disclosed together in sketched diagrams 10-12 for same values of $t$ and material parameters, it represented clearly from plotted Figs. 10, 11 and 12 that as anticipated the simple Newtonian fluid is more rapidly grow and fractionalized Jeffrey fluid is slowly grow. It is clearly seen that the ordinary and fractionalized Jeffrey fluids at different values of $\theta$, represents the ordinary Jeffrey is moved faster than fractionalized Jeffrey fluid. It can also be described from these figures that for highest values of slip parameter the four fluid models are decelerated as expected. In the making of all graphs, we used SI units and all graphs are prepared on Mathcad software and then using paint and Inkscape, we obtained the final form of the graphs.

Acknowledgement: The authors, Dr. Muhammad Jamil and Mr. Abdul Haleem, are thoroughly obliged to the De- 


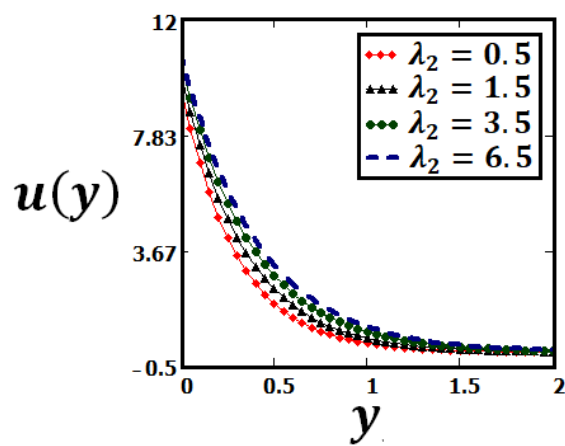

(a)

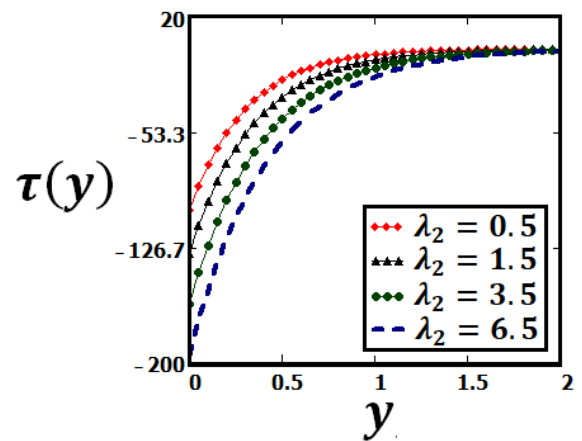

(b)

Figure 4: Profiles of the velocity field $u(y, t)$ and the shear stress $\tau(y, t)$ for fractionalized Jeffrey fluid given by Eqs. (16) and (21), for $A=$ $1, \quad v=1.427, \mu=19, \lambda_{1}=9, \quad H=0.5, K=2, \alpha=0.5, \theta=0.2, p=4, t=2 s$ and different values of $\lambda_{2}$.

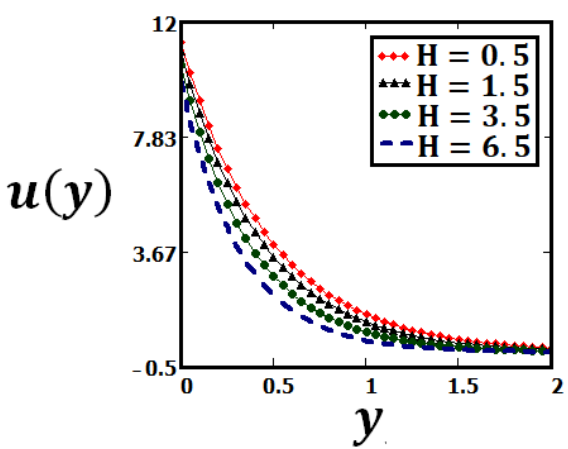

(a)

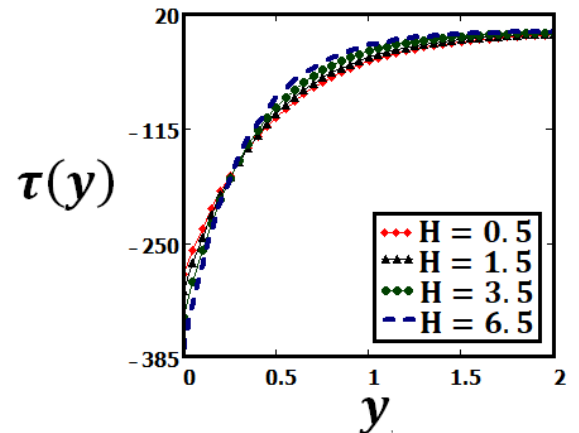

(b)

Figure 5: Profiles of the velocity field $u(y, t)$ and the shear stress $\tau(y, t)$ for fractionalized Jeffrey fluid given by Eqs. (16) and (21), for $A=$ $1, \quad v=1.427, \mu=19, \lambda_{1}=3, \lambda_{2}=2, K=2, \alpha=0.5, \theta=0.2, p=4, t=2 s$ and different values of $H$.

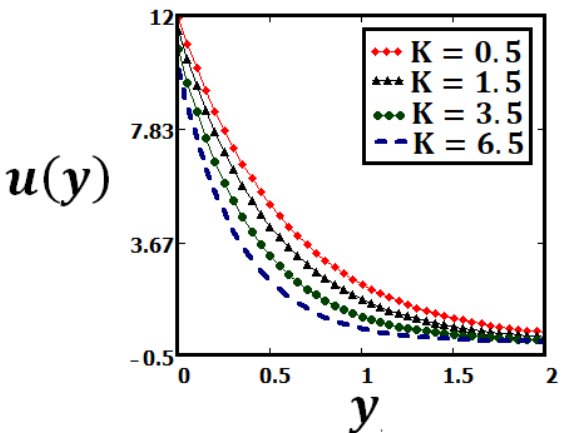

(a)

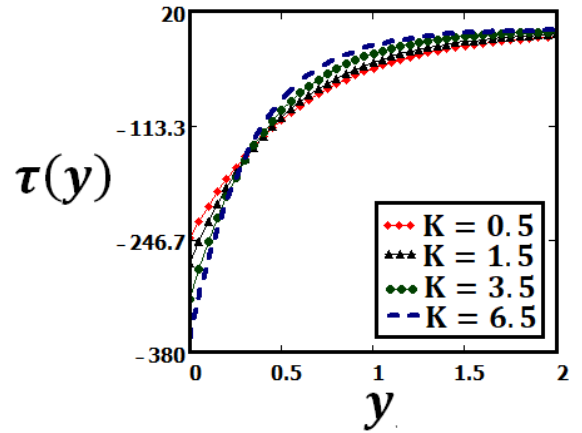

(b)

Figure 6: Profiles of the velocity field $u(y, t)$ and the shear stress $\tau(y, t)$ for fractionalized Jeffrey fluid given by Eqs. (16) and (21), for $A=$ $1, \quad v=1.427, \mu=19, \lambda_{1}=3, \lambda_{2}=2, H=0.5, \alpha=0.5, \theta=0.2, p=4, t=2 s$ and different values of $K$. 


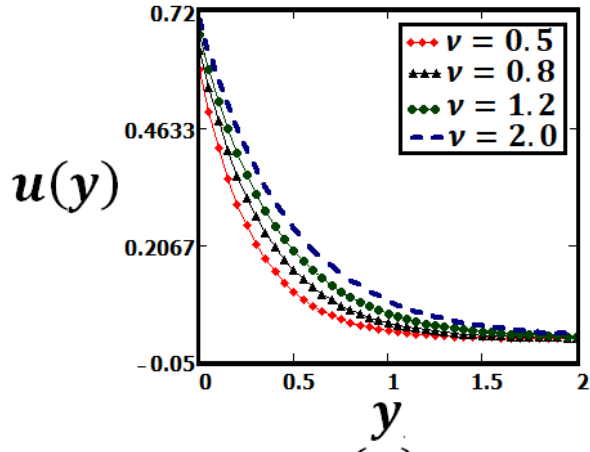

(a)

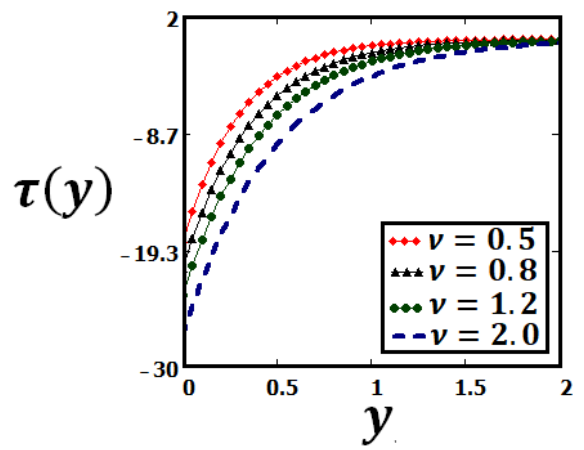

(b)

Figure 7: Profiles of the velocity field $u(y, t)$ and the shear stress $\tau(y, t)$ for fractionalized Jeffrey fluid given by Eqs. (16) and (21), for $A=$ 1, $\rho=13.313, \lambda_{1}=3, \lambda_{2}=2, H=0.5, K=2, \alpha=0.5, \theta=0.2, p=4, t=1 s$ and different values of $v$.

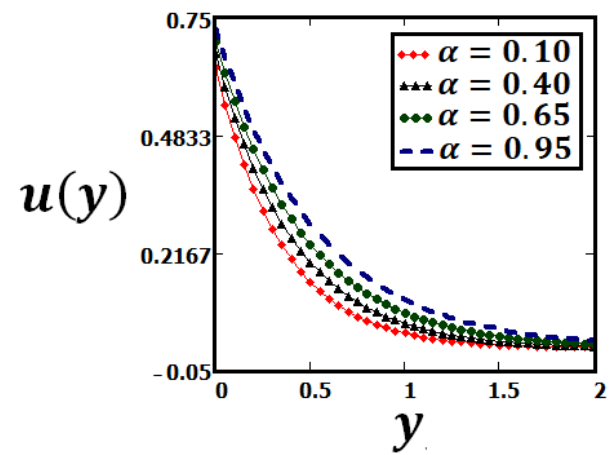

(a)

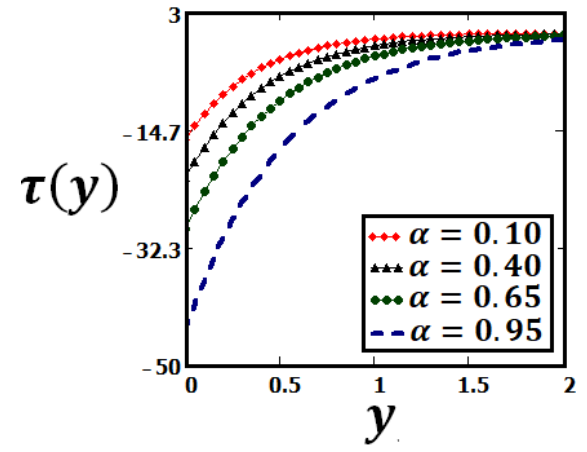

(b)

Figure 8: Profiles of the velocity field $u(y, t)$ and the shear stress $\tau(y, t)$ for fractionalized Jeffrey fluid given by Eqs. (16) and (21), for $A=$ $1, \quad v=1.427, \mu=19, \lambda_{1}=3, \lambda_{2}=2, H=0.5, K=2, \theta=0.2, p=4, t=1 s$ and different values of $\alpha$.

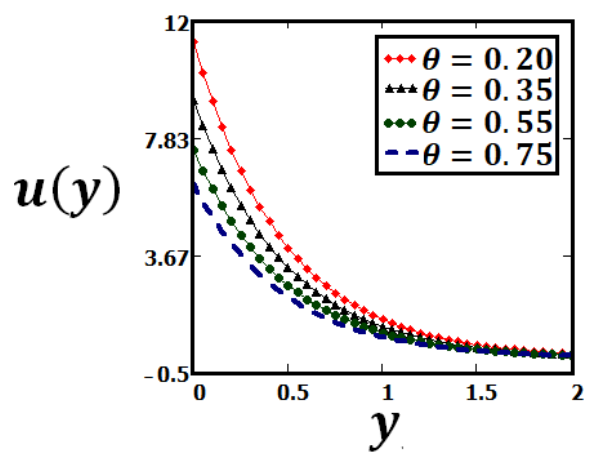

(a)

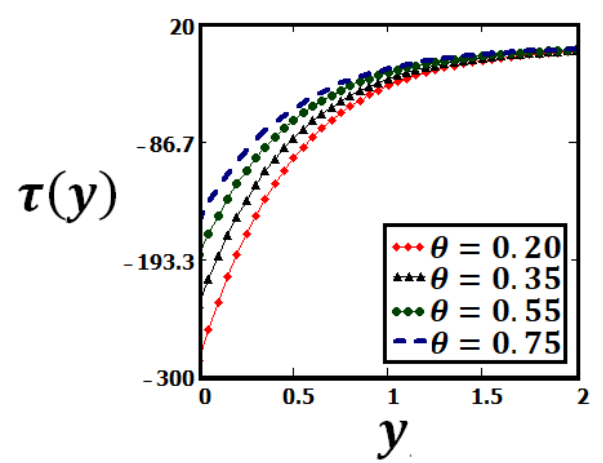

(b)

Figure 9: Profiles of the velocity field $u(y, t)$ and the shear stress $\tau(y, t)$ for fractionalized Jeffrey fluid given by Eqs. (16) and (21), for $A=$ $1, \quad v=1.427, \mu=19, \lambda_{1}=3, \lambda_{2}=2, H=0.5, K=2, \alpha=0.5, p=4, t=2 s$ and different values of $\theta$. 

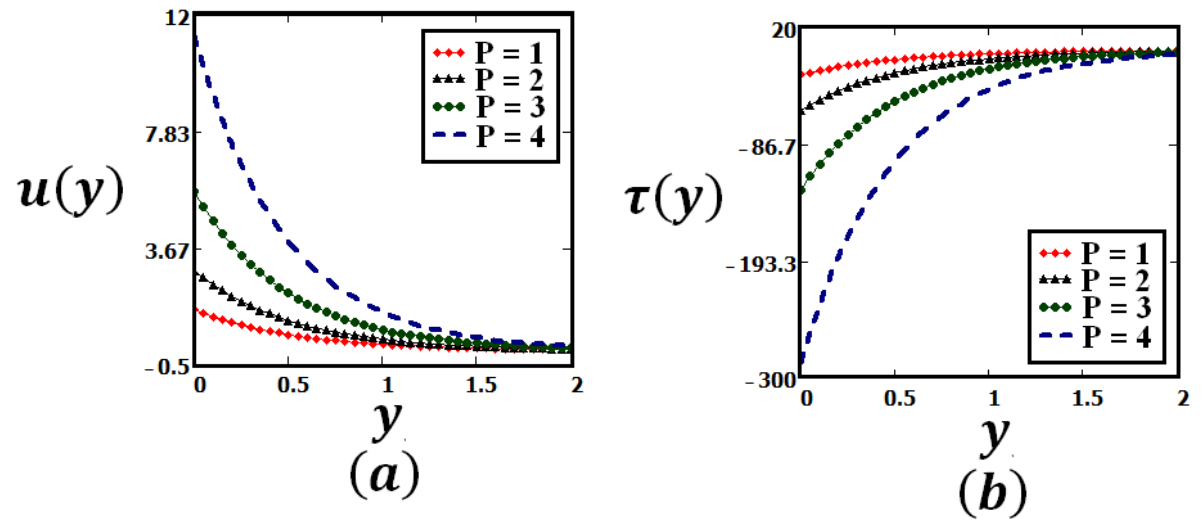

Figure 10: Profiles of the velocity field $u(y, t)$ and the shear stress $\tau(y, t)$ for fractionalized Jeffrey fluid given by Eqs. (16) and (21), for $A=$ $1, \quad v=1.427, \mu=19, \lambda_{1}=3, \lambda_{2}=2, \quad H=0.5, K=2, \alpha=0.5, \theta=0.2, \quad t=2 s$ and different values of $p$.

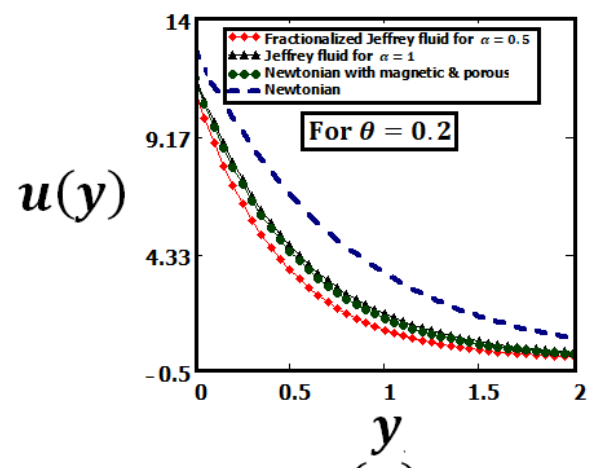

(a)

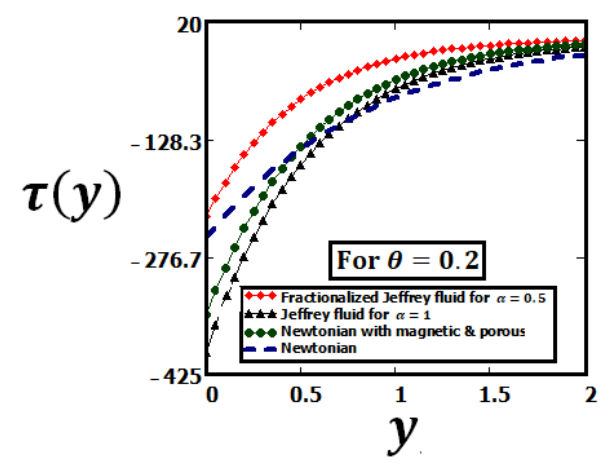

(b)

Figure 11: Profiles of the velocity field $u(y, t)$ and the shear stress $\tau(y, t)$ for fractionalized \& ordinary Jeffrey fluid given by Eqs. (16) and (21), Newtonian fluid with MHD \& parous and Newtonian fluid given by Eqs. (32) and (33) for $A=1, \quad v=1.427, \mu=19, \lambda_{1}=3, \lambda_{2}=2, \quad H=$ $0.5, K=2, \alpha=0.5, \theta=0.2, p=4$ and $t=2 s$.

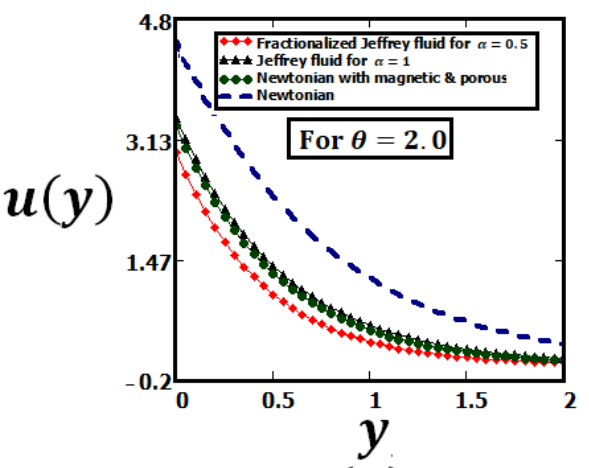

(a)

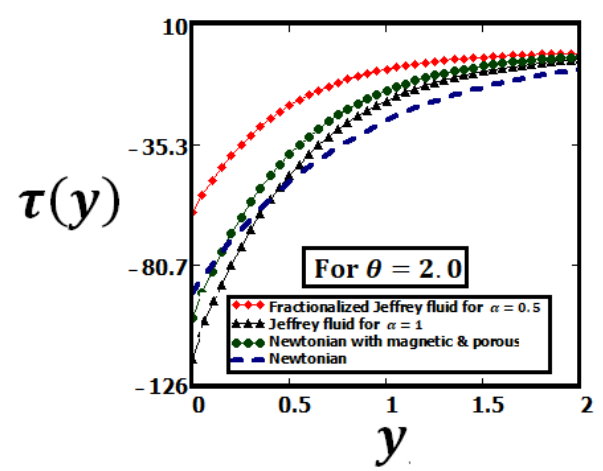

(b)

Figure 12: Profiles of the velocity field $u(y, t)$ and the shear stress $\tau(y, t)$ for fractionalized \& ordinary Jeffrey fluid given by Eqs. (16) and (21), Newtonian fluid with MHD \& parous and Newtonian fluid given by Eqs. (32) and (33) for $A=1, \quad v=1.427, \mu=19, \lambda_{1}=3, \lambda_{2}=2, H=$ $0.5, K=2, \alpha=0.5, \theta=2, p=4$ and $t=2 \mathrm{~s}$. 

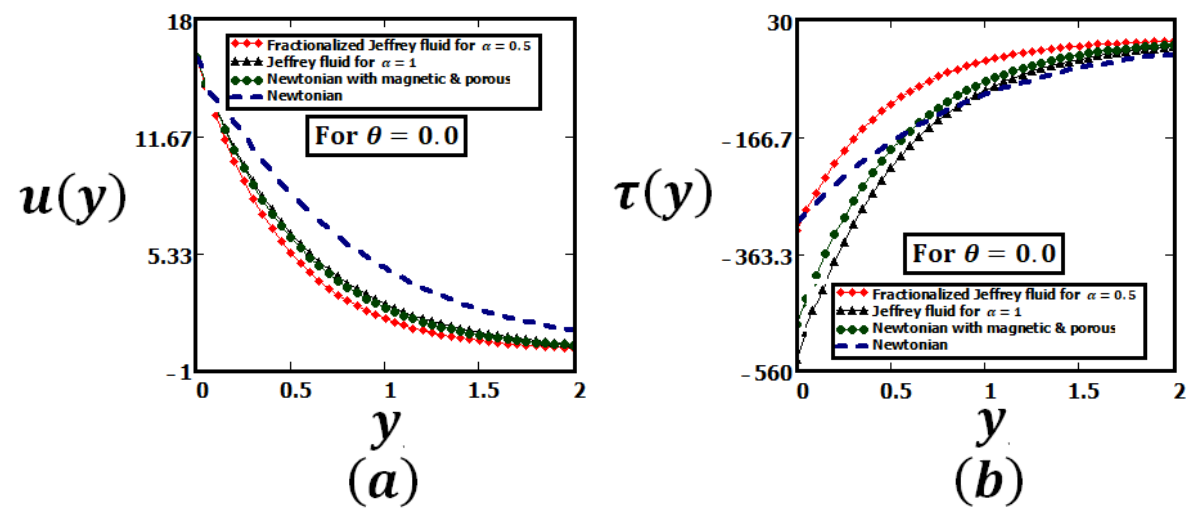

Figure 13: Profiles of the velocity field $u(y, t)$ and the shear stress $\tau(y, t)$ for fractionalized \& ordinary Jeffrey fluid given by Eqs. (16) and (21), Newtonian fluid with MHD \& parous and Newtonian fluid given by Eqs. (32) and (33) for $A=1, \quad v=1.427, \mu=19, \lambda_{1}=3, \lambda_{2}=2, H=$ $0.5, K=2, \alpha=0.5, \theta=0.0, p=4$ and $t=2 s$.

partment of Mathematics, NED University of Engineering \& Technology, Karachi-75270, Pakistan and also would like to express their gratitude to Higher Education Commission of Pakistan for assisting and facilitating this research work.

\section{References}

[1] Khan A., Zaman G., Ahmad S., Chohan M.I., Some Exact Solutions of Generalized Jeffrey Fluid Using N-Transform, Amer. J. Comput. Math., 2017, 7(04), 402.

[2] Han S., Zheng L., Zhang X., Slip effects on a generalized Burgers fluid flow between two side walls with fractional derivative, J. Egypt. Math. Soc., 2016, 24(1), 130-7.

[3] Jamil M., Effects of slip on oscillating fractionalized Maxwell fluid, Nonlin. Eng., 2016, 5(1), 25-36.

[4] Khan M., Maqbool K., Hayat T., Influence of Hall current on the flows of a generalized Oldroyd-B fluid in a porous space, Act. Mechan., 2006, 184(1-4), 1-3.

[5] Hayat T., Waqas M., Shehzad S.A., Alsaedi A., MHD stagnation point flow of Jeffrey fluid by a radially stretching surface with viscous dissipation and Joule heating, J. Hydrol. Hydromech., 2015, 63(4), 311-7.

[6] Khan M., Partial slip effects on the oscillatory flows of a fractional Jeffrey fluid in a porous medium, J. Por. Media, 2007, 10(5).

[7] Jakati S.V., Raju B.T., Nargund A.L., Sathyanarayana S.B., The Effect of Brownian motion and thermophoresis on nanofluids stretching for Jaffrey fluid model, Int. J. Latest Transac. Eng. Sci., 2018, 3(3), 1-9.

[8] Shehzad S.A., Hayat T., Alhuthali M.S., Asghar S., MHD threedimensional flow of Jeffrey fluid with Newtonian heating, J. Centr. South Univ., 2014, 21(4), 1428-33.

[9] Sultan F., Khan N.A., Qasim M., Afridi M.I., Numerical Simulation of the Flow of Nano-Eyring-Powell Fluid through a Curved Artery with Time-Variant Stenosis and Aneurysm, Nihon Re- oroji Gakkaishi, 2019, 47(2), 75-85.

[10] Raju K., Parandhama A., Raju M.C., Ramesh Babu K., Unsteady MHD free convection Jeffery fluid flow of radiating and reacting past a vertical porous plate in Slip-flow regime with heat source, Front. Heat Mass Transf., 2018, 10.

[11] Nadeem S., Hussain A., Majid K., Stagnation flow of a Jeffrey fluid over a shrinking sheet, Zeitschrift für Naturforschung A., 2010, 65(6-7), 540-8.

[12] Farooq M., Gull N., Alsaedi A., Hayat T., MHD flow of a Jeffrey fluid with Newtonian heating, J. Mechanic., 2015, 31(3), 31929.

[13] Ara A., Khan N.A., Sultan F., Ullah S., Numerical simulation of Jeffery-Hamel flow bingham plastic fluid and heat transfer in the presence of magnetic field, Appl. Compution. Math., 2019, 18(2), 135-48.

[14] Rao A.S., Nagendra N., Prasad V.R., Heat transfer in a nonNewtonian Jeffrey's fluid over a non-isothermal wedge, Proc. Eng., 2015, 127, 775-82.

[15] Hayat T., Rafiq M., Ahmad B., Soret and Dufour effects on MHD peristaltic flow of Jeffrey fluid in a rotating system with porous medium, PloS One, 2016, 11(1).

[16] Tripathi D., Ali N., Hayat T., Chaube M.K., Hendi A.A., Peristaltic flow of MHD Jeffrey fluid through finite length cylindrical tube, Appl. Math. Mechanic., 2011, 32(10), 1231.

[17] Das K., Influence of slip and heat transfer on MHD peristaltic flow of a Jeffrey fluid in an inclined asymmetric porous channel, Ind. J. Math., 2012, 54(1), 19-45.

[18] Afridi M.I., Tlili I., Goodarzi M., Osman M., Khan N.A., Irreversibility analysis of hybrid nanofluid flow over a thin needle with effects of energy dissipation, Symmetry, 2019, 11(5), 663.

[19] Jamil M., Khan A.N., Shahid N., Fractional magnetohydrodynamics Oldroyd-B fluid over an oscillating plate, Therm. Sci., 2013, 17(4), 997-1011.

[20] Mohanty J., Das J.K., Mishra S.R., Chemical reaction effect on MHD jeffery fluid over a stretching sheet with heat generation/absorption, Series Modell B, 2014, 83, 1-7.

[21] Kashyap K.P., Ojjela O., Das S.K., MHD slip flow of chemically reacting UCM fluid through a dilating channel with heat source/sink, Nonlin. Eng., 2019, 8(1), 523-33. 
[22] Hayat T., Sajjad R., Asghar S., Series solution for MHD channel flow of a Jeffery fluid, Commun. Nonlin. Sci. Numer. Simulat., 2010, 15(9), 2400-6.

[23] Zheng L., Liu Y., Zhang X., Slip effects on MHD flow of a generalized Oldroyd-B fluid with fractional derivative, Nonlin. Analys.: Real World Appl., 2012, 13(2), 513-23.

[24] Sharma K., Gupta S., Viscous dissipation and thermal radiation effects in MHD flow of Jeffrey nanofluid through impermeable surface with heat generation/absorption, Nonlin. Eng., 2017, 6(2), 153-66.

[25] Jamil M., Khan N.A., Slip effects on fractional viscoelastic fluids, Intern. J. Different. Equat., 2011, 2011.

[26] Liu Y., Guo B., Effects of second-order slip on the flow of a fractional Maxwell MHD fluid, J. Assoc. Arab Univ. Basic Appl. Sci., 2017, 24, 232-41.

[27] Amanulla C.H., Wakif A., Boulahia Z., Fazuruddin S., Mohammed S.N., A study on non-Newtonian transport phenomena in MHD fluid flow from a vertical cone with Navier slip and convective heating, Nonlin. Eng., 2019, 8(1), 534-45.
[28] Iftikhar N., Baleanu D., Husnine S.M., Shabbir K., Magnetohydrodynamic mixed convection flow of Jeffery fluid with thermophoresis, Soret and Dufour effects and convective condition, AIP Adv., 2019, 9(3), 035251.

[29] Mainardi F., Fractional calculus and waves in linear viscoelasticity: An introduction to mathematical models, Imper. College Press, 2010.

[30] Mathai A.M., Saxena R.K., Haubold H.J., The H-Functions: Theory and Applications, Springer, New York, 2010.

[31] Podlubny I., Fractional Differential Equations, Acad. press, 1999.

[32] Mainardi F., Fractional calculus and waves in linear viscoelasticity: An introduction to mathematical models, Imper. College Press, 2010. 